\title{
FORMALIZATION OF ABSTRACT STATE TRANSITION SYSTEMS FOR SAT
}

\author{
FILIP MARIĆ AND PREDRAG JANIČIĆ
}

Faculty of Mathematics, University of Belgrade, Studentski Trg 16, 11000 Belgrade

e-mail address: filip@matf.bg.ac.rs, janicic@matf.bg.ac.rs

\begin{abstract}
We present a formalization of modern SAT solvers and their properties in a form of abstract state transition systems. SAT solving procedures are described as transition relations over states that represent the values of the solver's global variables. Several different SAT solvers are formalized, including both the classical DPLL procedure and its state-of-the-art successors. The formalization is made within the Isabelle/HOL system and the total correctness (soundness, termination, completeness) is shown for each presented system (with respect to a simple notion of satisfiability that can be manually checked). The systems are defined in a general way and cover procedures used in a wide range of modern SAT solvers. Our formalization builds up on the previous work on state transition systems for SAT, but it gives machine-verifiable proofs, somewhat more general specifications, and weaker assumptions that ensure the key correctness properties. The presented proofs of formal correctness of the transition systems can be used as a key building block in proving correctness of SAT solvers by using other verification approaches.
\end{abstract}

\section{INTRODUCTION}

The problem of checking propositional satisfiability (SAT) is one of the central problems in computer science. It is the problem of deciding if there is a valuation of variables under which a given propositional formula (in conjunctive normal form) is true. SAT was the first problem that was proved to be NP-complete Coo71 and it still holds a central position in the field of computational complexity. SAT solvers, procedures that solve the SAT problem, are successfully used in many practical applications such as electronic design automation, software and hardware verification, artificial intelligence, and operations research.

Most state-of-the-art complete SAT solvers are essentially based on a branch and backtrack procedure called Davis-Putnam-Logemann-Loveland or the DPLL procedure DP60, DLL62. Modern SAT solvers usually also employ (i) several conceptual, high-level algorithmic additions to the original DPLL procedure, (ii) smart heuristic components, and (iii) better low-level implementation techniques. Thanks to these, spectacular improvements in the performance of SAT solvers have been achieved and nowadays SAT solvers can decide satisfiability of CNF formulae with tens of thousands of variables and millions of clauses.

1998 ACM Subject Classification: F.3.1, F.4.1.

Key words and phrases: formal verification, SAT solving, abstract state transition systems, Isabelle/HOL.

LOGICAL METHODS IN COMPUTER SCIENCE
DOI:10.2168/LMCS-7 (3:19) 2011
(C) F. Marić and P. Janičić (c) Creative Commons 
The tremendous advance in the SAT solving technology has not been accompanied with corresponding theoretical results about the solver correctness. Descriptions of new procedures and techniques are usually given in terms of implementations, while correctness arguments are either not given or are given only in outlines. This gap between practical and theoretical progress needs to be reduced and first steps in that direction have been made only recently, leading to the ultimate goal of having modern SAT solvers that are formally proved correct. That goal is vital since SAT solvers are used in applications that are very sensitive (e.g., software and hardware verification) and their misbehaviour could be both financially expensive and dangerous from the aspect of security. Ensuring trusted SAT solving can be achieved by two approaches.

One approach for achieving a higher level of confidence in SAT solvers' results, successfully used in recent years, is proof-checking [ZM03, GN03, Gel07, WA09, DFMS10]. In this approach, solvers are modified so that they output not only sat or unsat answers, but also justification for their claims (models for satisfiable instances and proof objects for unsatisfiable instances) that are then checked by independent proof-checkers. Proofchecking is relatively easy to implement, but it has some drawbacks. First, justification for every solved SAT instance has to be verified separately. Also, generating unsatisfiability proofs introduces some overhead to the solver's running time, proofs are typically large and may consume gigabytes of storage space, and proof-checking itself can be time consuming Gel07. Since proof-checkers have to be trusted, they must be very simple programs so they can be "verified" by code inspection 1 . On the other hand, in order to be efficient, they must use specialized functionality of the underlying operating system which reduces the level of their reliability (e.g., the proof checker used in the SAT competitions uses Linux's mmap functionality [Gel07]).

The other approach for having trusted solvers' results is to verify the SAT solver itself, instead of checking each of its claims. This approach is very demanding, since it requires formal analysis of the complete solver's behaviour. In addition, whenever the implementation of the solver changes, the correctness proofs must be adapted to reflect the changes. Still, in practice, the core solving procedure is usually stable and stays fixed, while only heuristic components frequently change. The most challenging task is usually proving the correctness of the core solving procedures, while heuristic components only need to satisfy relatively simple properties that are easily checked. This approach gives also the following benefits:

- Although the overheads of generating unsatisfiability proofs during solving are not unmanageable, in many applications they can be avoided if the solver itself is trusted 2

- Verification of modern SAT solvers could help in better theoretical understanding of how and why they work. A rigorous analysis and verification of modern SAT solvers may reveal some possible improvements in underlying algorithms and techniques which can influence and improve other solvers as well.

- Verified SAT solvers can serve as trusted kernel checkers for verifying results of other untrusted verifiers such as BDDs, model checkers, and SMT solvers. Also, verification of some SAT solver modules (e.g., Boolean constraint propagation) can serve as a basis for creating both verified and efficient proof-checkers for SAT.

\footnotetext{
${ }^{1}$ Alternatively, proof-checkers could be formally verified by a proof assistant, and then their correctness would rely on the correctness of the proof assistant.

${ }^{2}$ In some applications, proofs of unsatisfiability are still necessary as they are used, for example, for extracting unsatisfiable cores and interpolants.
} 


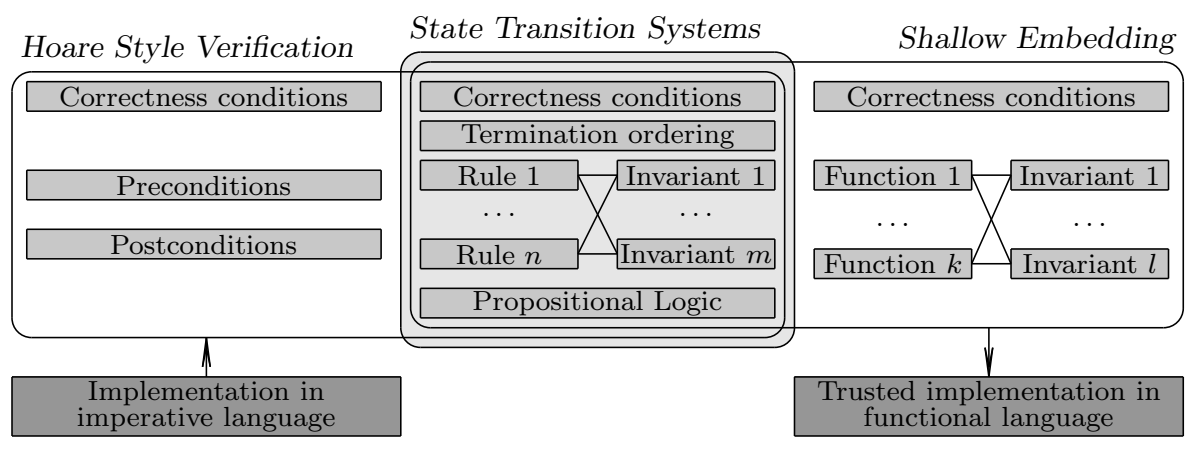

Figure 1: Different approaches for SAT solver verification

In order to prove correctness of a SAT solver, it has to be formalized in some meta-theory so its properties can be analyzed in a rigorous mathematical manner. In order to achieve the desired highest level of trust, formalization in a classical "pen-and-paper" fashion is not satisfactory and, instead, a mechanized and machine-checkable formalization is preferred. The formal specification of a SAT solver can be made in several ways (illustrated in Figure 1, each with an appropriate verification paradigm and each having its own advantages and disadvantages, described in the following text).

Verification of abstract state transition system: State transition systems are an abstract and purely mathematical way of specifying program behaviour. Using this approach, the SAT solver's behaviour is modelled by transitions between states that represent the values of the solver's global variables. Transitions can be made only by following precisely defined transition rules. Proving correctness of state transition systems can be performed by the standard mathematical apparatus. There are state transition systems describing the top-level architecture of the modern DPLL-based SAT solvers (and related SMT solvers) [KG07, NOT06] and their correctness has been informally shown.

The main advantage of the abstract state transition systems is that they are mathematical objects, so it is relatively easy to make their formalization within higher-order logic and to formally reason about them. Also, their verification can be a key building block for other verification approaches. Disadvantages are that the transition systems do not specify many details present in modern solver implementations and that they are not directly executable.

Verified implementation within a proof assistant: A program's behaviour can be specified within the higher-order logic of a proof assistant (regarded as a purely functional programming language). This approach is often called shallow embedding into HOL. Specifications may vary from very abstract ones to detailed ones covering most details present in the real SAT solver's code. The level of details can incrementally be increased (e.g., by using a datatype refinement). Having the specification inside the logic, its correctness can be proved again by using the standard mathematical apparatus (mainly induction and equational reasoning). Based on the specification, executable functional programs can be generated by means of code extraction - the term language of the logic within the proof assistant is identified with the term language of the target language and the verified program correctness is transferred to the exported program, up to simple transformation rules. 
Advantages of using the shallow embedding are that, once the solver is defined within the proof assistant, it is possible to verify it directly inside the logic and a formal model of the operational or denotational semantics of the language is not required. Also, extracted executable code can be trusted with a very high level of confidence. On the other hand, the approach requires building a fresh implementation of a SAT solver within the logic. Also, since higher-order logic is a pure functional language, it is unadapted to modelling imperative data-structures and their destructive updates. Special techniques must be used to have mutable data-structures and, consequently, an efficient generated code $\mathrm{BKH}^{+} 08$.

Verification of the real implementations: The most demanding approach for verifying a SAT solver is to directly verify the full real-world solver code. Since SAT solvers are usually implemented in imperative programming languages, verifying the correctness of implementation can be made by using the framework of Hoare logic [Hoa69] — a formal system for reasoning about programs written in imperative programming languages. The program behaviour can then be described in terms of preconditions and postconditions for pieces of code. Proving the program correctness is made by formulating and proving verification conditions. For instance, Isabelle/HOL provides a formal verification environment for sequential imperative programs ([Sch06]).

The main benefit of using the Hoare style verification is that it enables reasoning about the imperative code, which is the way that most real-world SAT solvers are implemented. However, since real code is overwhelmingly complex, simpler approximations are often made and given in pseudo-programming languages. This can significantly simplify the implementation, but leaves a gap between the correctness proof and the real implementation.

In this paper we focus on the first verification approach as it is often suitable to separate the verification of the abstract algorithms and that of their specific implementations 3 In addition, state transition systems, as the most abstract specifications, cover the widest range of existing SAT solver implementations. Moreover, the reasoning used in verifying abstract state transition systems for SAT can serve as a key building block in verification of more detailed descriptions of SAT solvers using the other two approaches described above (as illustrated by Figure 1). Indeed, within our SAT verification project [MJ09], we have already applied these two approaches [Mar09, Mar10, MJ10, and in both cases the correctness arguments were mainly reduced to correctness of the corresponding abstract state transition systems. These transition systems and their correctness proofs are presented in this paper for the first time, after they evolved to some extent through application within the other two verification approaches.

The methodology that we use in this paper for the formalization of SAT solvers via transition systems is incremental refinement: the formalization begins with a most basic specification, which is then refined by introducing more advanced techniques, while preserving the correctness. This incremental approach proves to be a very natural approach in formalizing complex software systems. It simplifies understanding of the system and reduces the overall verification effort. Each of the following sections describes a separate abstract state transition system. Although, formally viewed, all these systems are independent, each new system extends the previous one and there are tight connections between

\footnotetext{
${ }^{3} \mathrm{~A}$ recent example is the $\mathrm{L} 4$ verified OS kernel, where a shallowly embedded Haskell specification of the kernel is verified, and then the $\mathrm{C}$ code is shown to implement the Haskell specification, yielding a natural separation of concepts and issues Kle10.
} 
them. Therefore, we do not expose each new system from scratch, but only give additions to the previous one. We end up with a system that rather precisely describes modern SAT solvers, including advanced techniques such as backjumping, learning, conflict analysis, forgetting and restarting. The systems presented are related to existing solvers, their abstract descriptions and informal correctness proofs.

The paper is accompanied by a full formalization developed within the Isabelle/HOL proof assistant 4 The full version of the paper ${ }^{5}$ contains an appendix with informal proofs of all lemmas used. All definitions, lemmas, theorems and proofs of top-level statements given in the paper correspond to their Isabelle counterparts, and here are given in a form accessible not only to Isabelle users, but to a wider audience.

The main challenge in each large formalization task is to define basic relevant notions in appropriate terms, build a relevant theory and a suitable hierarchy of lemmas that facilitates constructing top-level proofs. Although in this paper we do not discuss all decisions made in the above directions, the final presented material is supposed to give the main motivating ideas and, implicitly, to illustrate a proof management technology that was used. The main purpose of the paper is to give a clear picture of central ideas relevant for verification of SAT transition systems, hopefully interesting both to SAT developers and to those involved in formalization of mathematics.

The main contributions of this paper are the following.

- SAT solving process is introduced by a hierarchical series of abstract transition systems, ending up with the state-of-the-art system.

- Formalization and mechanical verification of properties of the abstract transition systems for SAT are performed (within this, invariants and well-founded relations relevant for termination are clearly given; conditions for soundness, completeness, and termination are clearly separated). Taking advantage of this formalization, different real-world SAT solvers can be verified, using different verification approaches.

- First proofs (either informal or formal) of some properties of modern SAT solvers (e.g., termination condition for frequent restarting) are given, providing deeper understanding of the solving process.

The rest of the paper is organized as follows: In Section 2 some background on SAT solving, abstract state transition systems, and especially abstract state transition systems for SAT is given. In Section 3 basic definitions and examples of propositional logic and CNF formulae are given. In Section 4, a system corresponding to basic DPLL search is formalized. In Section 5, that system is modified and backtracking is replaced by more advanced backjumping. In Section 6 , the system is extended by clause learning and forgetting. In Section 7 and Section 8 a system with conflict analysis and a system with restarting and forgetting are formalized. In Section 9 we discuss related work and our contributions. In Section 10, final conclusions are drawn.

\section{BACKGROUND}

In this section we give a brief, informal overview of the SAT solving process, abstract state transition systems and abstract state transition systems for SAT. The paper does

\footnotetext{
${ }^{4}$ The whole presented formalization is available from AFP Mar08 and, the latest version, from http://argo.matf.bg.ac.rs.

${ }^{5}$ The full version of the paper is available from http://argo.matf.bg.ac.rs
} 
not intend to be a tutorial on modern DPLL-based SAT solving techniques - the rest of the paper contains only some brief explanations and assumes the relevant background knowledge (more details and tutorials on modern SAT solving technology can be found in other sources e.g., BHMW09, Mar09]).

2.1. SAT Solving. SAT solvers are decision procedures for the satisfiability problem for propositional formulae in conjunctive normal form (CNF). State-of-the-art SAT solvers are mainly based on a branch-and-backtrack procedure called DPLL (Davis-Putnam-LogemannLoveland) DP60, DLL62 and its modern successors. The original DPLL procedure (shown in Figure 2) combines backtrack search with some basic, but efficient inference rules.

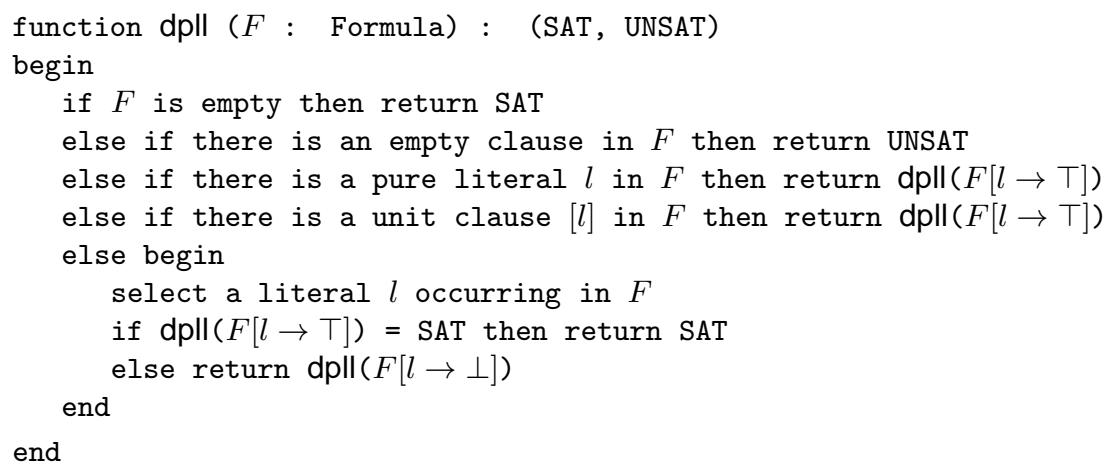

Figure 2: The original DPLL procedure

The search component selects a branching literal $l$ occurring in the formula $F$, and tries to satisfy the formula obtained by replacing $l$ with $T$ and simplifying afterwards. If the simplified formula is satisfiable, so is the original formula $F$. Otherwise, the formula obtained from $F$ by replacing $l$ with $\perp$ and by simplifying afterwards is checked for satisfiability and it is satisfiable if and only if the original formula $F$ is satisfiable. This process stops if the formula contains no clauses or if it contains an empty clause. A very important aspect of the search process is the strategy for selecting literals for branching - while not important for the correctness of the procedure, this strategy can have a crucial impact on efficiency.

The simple search procedure is enhanced with several simple inference mechanisms. The unit clause rule is based on the fact that if there is a clause with a single literal present in $F$, its literal must be true in order to satisfy the formula (so there is no need for branching on that literal). The pure literal rule is based on the fact that if a literal occurs in the formula, but its opposite literal does not, if the formula is satisfiable, in one of its models that literal is true. These two rules are not necessary for completeness, although they have a significant impact on efficiency.

Passing valuations instead of modifying the formula. In the original DPLL procedure, the formula considered is passed as a function argument, and modified throughout recursive calls. This is unacceptably inefficient for huge propositional formulae and can be replaced by a procedure that maintains a current (partial) valuation $M$ and, rather than modifying the formula, keeps the formula constant and checks its value against the current valuation (see Figure 3). The inference rules used in the original procedure must be adapted to fit this variant of the algorithm. The unit clause rule then states that if there is a clause in $F$ such that all its literals, except exactly one, are false in $M$, and that literal is undefined 
in $M$, then this literal must be added to $M$ in order to satisfy this clause. The pure literal rule turns out to be too expensive in this context, so modern solvers typically do not use it.

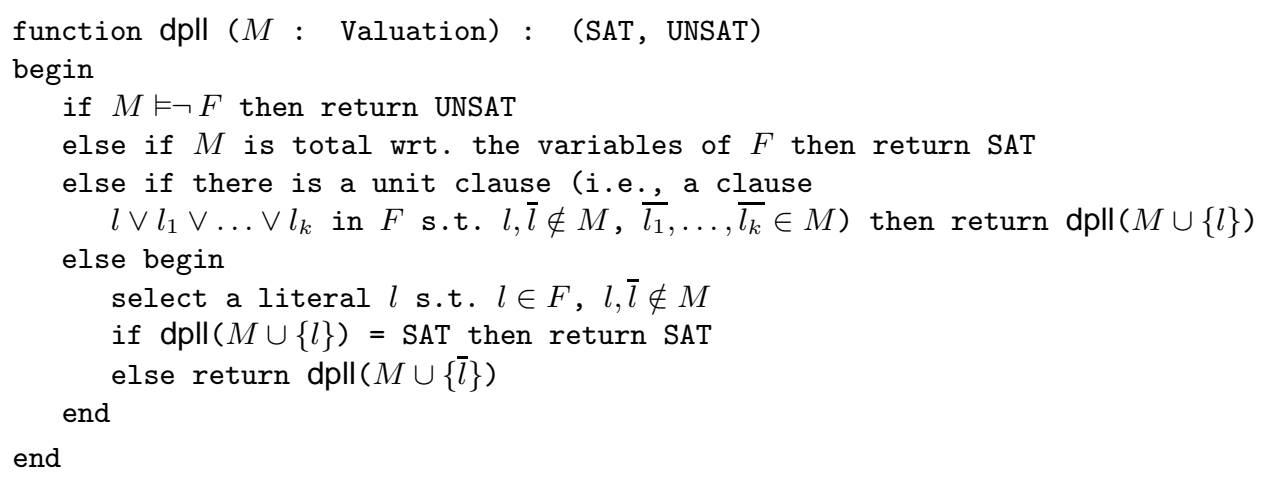

Figure 3: DPLL procedure with valuation passing

Non-recursive implementation. To gain efficiency, modern SAT solvers implement DPLL-like procedures in a non-recursive fashion. Instead of passing arguments through recursive calls, both the current formula $F$ and the current partial valuation $M$ are kept as global objects. The valuation acts as a stack and is called assertion trail. Since the trail represents a valuation, it must not contain repeated nor opposite literals (i.e., it is always distinct and consistent). Literals are added to the stack top (asserting) or removed from the stack top (backtracking). The search begins with an empty trail. During the solving process, the solver selects literals undefined in the current trail $M$ and asserts them, marking them as decision literals. Decision literals partition the trail into levels, and the level of a literal is the number of decision literals that precede that literal in the trail. After each decision, unit propagation is exhaustively applied and unit literals are asserted to $M$, but as implied literals (since they are not arbitrary decisions). This process repeats until either (i) a clause in $F$ is found which is false in the current trail $M$ (this clause is called a conflict clause) or (ii) all the literals occurring in $F$ are defined in $M$ and no conflict clause is found in $F$. In the case (i), a conflict reparation (backtracking) procedure must be applied. In the basic variant of the conflict reparation procedure, the last decision literal $l$ and all literals after it are backtracked from $M$, and the opposite literal of $l$ is asserted, also as an implied literal. If there is no decision literal in $M$ when a conflict is detected, then the formula $F$ is unsatisfiable. In the case (ii), the formula is found to be satisfiable and $M$ is its model.

Modern DPLL enhancements. For almost half of a century, DPLL-based SAT procedures have undergone various modifications and improvements. Accounts of the evolution of SAT solvers can be found in recent literature [BHMW09, GKSS07]. Early SAT solvers based on DPLL include Tableau (NTAB), POSIT, 2cl and CSAT, among others. In the mid 1990's, a new generation of solvers such as GRASP [MSS99], SATO [Zha97], Chaff $\mathrm{MMZ}^{+}$01, and BerkMin GN02 appeared, and in these solvers a lot of attention was payed to optimisation of various aspects of the DPLL algorithm. Some influential modern SAT solvers include MiniSat [ES04] and PicoSAT Bie08].

A significant improvement over the basic search algorithm is to replace the simple conflict reparation based on backtracking by a more advanced one based on conflict driven backjumping, first proposed in the Constraint Satisfaction Problem (CSP) domain [BHZ06]. Once a conflict is detected, a conflict analysis procedure finds sequence of decisions (often 
buried deeper in the trail) that eventually led to the current conflict. Conflict analysis can be described in terms of graphs and the backjump clauses are constructed by traversing a graph called implication graph [MSS99]. The process can also be described in terms of resolution that starts from the conflict clause and continues with clauses that caused unit propagation of literals in that clause [ZM02]. There are several strategies for conflict analysis, leading to different backjump clauses [BHMW09]. Most conflict analysis strategies are based on the following scheme:

(1) Conflict analysis starts with a conflict clause (i.e., the clause from $F$ detected to be false in $M$ ). The conflict analysis clause $C$ is set to the conflict clause.

(2) Each literal from the current conflict analysis clause $C$ is false in the current trail $M$ and is either a decision literal or a result of a propagation. For each propagated literal $l$ it is possible to find a clause (reason clause) that caused $l$ to be propagated. The propagated literals from $C$ are then replaced (it will be said explained) by remaining literals from their reason clauses. The process of conflict analysis then continues.

The described procedure continues until some termination condition is met, and the backjump clause is then constructed. Thanks to conflict driven backjumping, a lot of unnecessary work can be saved compared to the simple backtrack operation. Indeed, the simple backtracking would have to consider all combinations of values for all decision literals between the backjump point and the last decision, while they are all irrelevant for the particular conflict.

The result of conflict analysis is usually a clause that is a logical consequence of $F$ and that explains a particular conflict that occurred. If this clause was added to $F$, then this type of conflict would occur never again during search (even in some other contexts, i.e., in some other parts of the search space). This is why solvers usually perform clause learning and append (redundant) deduced clauses to $F$. However, if the formula $F$ becomes too large, some clauses have to be forgotten. Conflict driven backjumping with clause learning were first incorporated into a SAT solver in the mid 1990's by Silva and Sakallah in GRASP [MSS99] and by Bayardo and Schrag in rel_sat [BS97]. DPLL-based SAT solvers employing conflict driven clause learning are often called $C D C L$ solvers.

Another significant improvement is to empty the trail and restart the search from time to time, in a hope that it would restart in an easier part of the search space. Randomized restarts were introduced by Gomes et al. GSK98 and further developed by Baptista and Marques-Silva [BMS00].

One of the most demanding operations during solving is the detection of false and unit clauses. Whenever a literal is asserted, the solver must check $F$ for their presence. To aid this operation, smart data structures with corresponding implementations are used. One of the most advanced ones is the two-watched literal scheme, introduced by Moskewicz et al. in their solver $\mathrm{zChaff}\left[\mathrm{MMZ}^{+} 01\right]$.

2.2. Abstract State Transition Systems. An abstract state transition system for an imperative program consists of a set of states $S$ describing possible values of the program's global variables and a binary transition relation $\rightarrow \subseteq S \times S$. The transition relation is usually the union of smaller transition relations $\rightarrow_{i}$, called the transition rules. If $s \rightarrow_{i} s^{\prime}$ holds, we say that the rule $i$ has been applied to the state $s$ and the state $s^{\prime}$ has been obtained. Transition rules are denoted as: 


$$
\text { Rulename: } \quad \frac{\text { cond }_{1} \ldots \operatorname{cond}_{k}}{\text { effect }}
$$

Above the line are the conditions $\operatorname{cond}_{1}, \ldots, \operatorname{cond}_{k}$ that the state $s$ must meet in order for the rule to be applicable and the effect denotes the effect that must be applied to the components of $s$ in order to obtain $s^{\prime}$.

More formally, transition rules can be defined as relations over states:

\section{Rulename $s s^{\prime}$ iff $\phi$}

where $\phi$ denotes a formula that describes conditions on $s$ that have to be met and the relationship between $s$ and $s^{\prime}$.

Some states are distinguished as initial states. An initial state usually depends on the program input. A state is a final state if no transition rules can be applied. Some states (not necessarily final) are distinguished as the outcome states carrying certain resulting information. If a program terminates in a final outcome state, it emits a result determined by this state. For a decision procedure (such as a SAT solver), there are only two possible outcomes: yes (sat) or no (unsat). A state transition system is considered to be correct if it has the following properties:

Termination: from each initial state $s_{0}$, the execution eventually reaches a final state (i.e., there are no infinite chains $s_{0} \rightarrow s_{1} \rightarrow \ldots$ ).

Soundness: the program always gives correct answers, i.e., if the program, starting with an input $I$ from an initial state $s_{0}$, reaches a final outcome state with a result $O$, then $O$ is the desired result for the input $I$.

Completeness: the program always gives an answer if it terminates, i.e., all final states are outcome states.

2.3. Abstract State Transition Systems for SAT. Two transition rule systems that model DPLL-based SAT solvers and related SMT solvers have been published recently. Both systems present a basis of the formalization described in this paper. The system of Krstić and Goel [KG07] gives a more detailed description of some parts of the solving process (particularly the conflict analysis phase) than the one given by Nieuwenhuis, Oliveras and Tinelli [NOT06], so we present its rules in Figure 4, In this system, along with the formula $F$ and the trail $M$, the state of the solver is characterized by the conflict analysis set $C$ that is either a set of literals (i.e., a clause) or the distinguished symbol no_cflct. Input to the system is an arbitrary set of clauses $F_{0}$. The solving starts from a initial state in which $F=F_{0}, M=[]$, and $C=$ no_cflct $_{\text {. }}$

The Decide rule selects a literal from a set of decision literals $L$ and asserts it to the trail as a decision literal. The set $L$ is typically just the set of all literals occurring in the input formulae. However, in some cases a smaller set can be used (based on some specific knowledge about the encoding of the input formula). Also, there are cases when this set is in fact larger than the set of all variables occurring in the input formula.6

The UnitPropag rule asserts a unit literal $l$ to the trail $M$ as an implied literal. This reduces the search space since only one valuation for $l$ is considered.

\footnotetext{
${ }^{6}$ For example, the standard DIMACS format for SAT requires specifying the number of variables and the clauses that make the formula, without guarantees that every variable eventually occurs in the formula.
} 


$$
\begin{aligned}
& \text { Decide : } \quad \frac{l \in L \quad l, \bar{l} \notin M}{M:=M l^{d}} \\
& \text { UnitPropag: } \quad \frac{l \vee l_{1} \vee \ldots \vee l_{k} \in F \quad \bar{l}_{1}, \ldots, \bar{l}_{k} \in M \quad l, \bar{l} \notin M}{M:=M l^{i}} \\
& \text { Conflict : } \quad \begin{array}{lll}
C=\text { no_cflct } & \bar{l}_{1} \vee \ldots \vee \bar{l}_{k} \in F & l_{1}, \ldots, l_{k} \in M \\
C:=\left\{l_{1}, \ldots, l_{k}\right\} &
\end{array} \\
& \text { Explain : } \quad \frac{l \in C \quad l \vee \bar{l}_{1} \vee \ldots \vee \bar{l}_{k} \in F \quad l_{1}, \ldots, l_{k} \prec l}{C:=C \cup\left\{l_{1}, \ldots, l_{k}\right\} \backslash\{l\}} \\
& \text { Learn : } \quad \frac{C=\left\{l_{1}, \ldots, l_{k}\right\} \quad \bar{l}_{1} \vee \ldots \vee \bar{l}_{k} \notin F}{F:=F \cup\left\{\bar{l}_{1} \vee \ldots \vee \bar{l}_{k}\right\}} \\
& \text { Backjump : } \quad \frac{C=\left\{l, l_{1}, \ldots, l_{k}\right\} \quad \bar{l} \vee \bar{l}_{1} \vee \ldots \vee \bar{l}_{k} \in F \quad \text { level } l>m \geq \text { level } l_{i}}{C:=\text { no_cflct }_{-} \quad M:=M^{[m]} \bar{l}^{i}} \\
& \text { Forget : } \quad \frac{C=n o_{-} c f l c t \quad c \in F \quad F \backslash c \vDash c}{F:=F \backslash c} \\
& \text { Restart : } \quad \frac{C=n o_{\_} \text {cflct }}{M:=M^{[0]}}
\end{aligned}
$$

Figure 4: Transition system for SAT solving by Krstić and Goel $\left(l_{i} \prec l_{j}\right.$ denotes that the literal $l_{i}$ precedes $l_{j}$ in $M, l^{d}$ denotes a decision literal, $l^{i}$ an implied literal, level $l$ denotes the decision level of a literal $l$ in $M$, and $M^{[m]}$ denotes the prefix of $M$ up to the level $m$ ).

The Conflict rule is applied when a conflict clause is detected. It initializes the conflict analysis and the reparation procedure, by setting $C$ to the set of literals of the conflict clause. This set is further refined by successive applications of the Explain rule, which essentially performs a resolution between the clause $C$ and the clause that is the reason of propagation of its literal $l$. During the conflict analysis procedure, the clause $C$ can be added to $F$ by the Learn rule. However, this is usually done only once - when there is exactly one literal in $C$ present at the highest decision level of $M$. In that case, the Backjump rule can be applied. That resolves the conflict by backtracking the trail to a level (usually the lowest possible) such that $C$ becomes unit clause with a unit literal $l$. In addition, unit propagation of $l$ is performed.

The Forget rule eliminates clauses. Namely, because of the learning process, the number of clauses in the current formula increases. When it becomes too large, detecting false and unit clauses becomes too demanding, so from time to time, it is preferable to delete from $F$ some clauses that are redundant. Typically, only learnt clauses are forgotten (as they are always redundant).

\section{Underlying TheOry}

As a framework of our formalization, higher-order logic is used, in a similar way as in the system Isabelle/HOL [NPW02]. Formulae and logical connectives of this logic $(\wedge, \vee, \neg, \longrightarrow$, $\longleftrightarrow)$ are written in the standard way. Equality is denoted by $=$. Function applications are 
written in prefix form, as in $\mathrm{f} x_{1} \ldots x_{n}$. Existential quantification is denoted by $\exists x . \ldots$ and universal quantification by $\forall x$. ....

In this section we will introduce definitions necessary for notions of satisfiability and notions used in SAT solving. Most of the definitions are simple and technical so we give them in a very dense form. They make the paper self-contained and can be used just for reference.

The correctness of the whole formalization effort eventually relies on the definition of satisfiable formulae, which is rather straightforward and easily checked by human inspection.

3.1. Lists, Multisets, and Relations. We assume that the notions of ordered pairs, lists and (finite) sets are defined within the theory. Relations and their extensions are used primarily in the context of ordering relations and the proofs of termination. We will use standard syntax and semantics of these types and their operations. However, to aid our formalization, some additional operations are introduced.

Definition 3.1 (Lists related).

- The first position of an element $e$ in a list $l$, denoted firstPos $e l$, is the zero-based index of the first occurrence of $e$ in $l$ if it occurs in $l$ or the length of $l$ otherwise.

- The prefix to an element $e$ of a list $l$, denoted by prefixTo $e l$, is the list consisting of all elements of $l$ preceding the first occurrence of $e$ (including $e$ ).

- The prefix before an element $e$ of a list $l$, denoted by prefixBefore $e l$ is the list of all elements of $l$ preceding the first occurrence of $e$ (not including $e$ ).

- An element $e_{1}$ precedes $e_{2}$ in a list $l$, denoted by $e_{1} \prec_{l} e_{2}$, if both occur in $l$ and the first position of $e_{1}$ in $l$ is less than the first position of $e_{2}$ in $l$.

- A list $p$ is a prefix of a list $l$ (denoted by $p \leq l$ ) if there exists a list $s$ such that $l=p @ s$.

Definition 3.2 (Multiset). A multiset over a type $X$ is a function $S$ mapping $X$ to natural numbers. A multiset is finite if the set $\{x \mid S(x)>0\}$ is finite. The union of multisets $S$ and $T$ is a function defined as $(S \cup T)(x)=S(x)+T(x)$.

Definition 3.3 (Relations related).

- The composition of two relations $\rho_{1}$ and $\rho_{2}$ is denoted by $\rho_{1} \circ \rho_{2}$. The $n$-th degree of the relation $\rho$ is denoted by $\rho^{n}$. The transitive closure of $\rho$ is denoted by $\rho^{+}$, and the transitive and reflexive closure of $\rho$ by $\rho^{*}$.

- A relation $\succ$ is well-founded iff:

$$
\forall P .((\forall x .(\forall y . x \succ y \longrightarrow P(y)) \longrightarrow P(x)) \longrightarrow(\forall x . P(x)))
$$

- If $\succ$ is a relation on $X$, then its lexicographic extension $\succ^{\text {lex }}$ is a relation on lists of $X$, defined by:

$$
\begin{aligned}
& s \succ^{\text {lex } t \quad \text { iff } \quad} \quad(\exists r . s=t @ r \wedge r \neq[]) \vee \\
&\left(\exists r s^{\prime} t^{\prime} a b . s=r @ a @ s^{\prime} \wedge t=r @ b @ t^{\prime} \wedge a \succ b\right)
\end{aligned}
$$

- If $\succ$ is a relation on $X$, then its multiset extension $\succ^{\text {mult }}$ is a relation defined over multisets over $X$ (denoted by $\left\langle x_{1}, \ldots, x_{n}\right\rangle$ ). The relation $\succ^{\text {mult }}$ is a transitive closure of the relation $\succ^{\text {mult }_{1}}$, defined by:

$$
\begin{aligned}
& S_{1} \succ^{\text {mult }_{1}} S_{2} \quad \text { iff } \quad \exists S S_{2}^{\prime} s_{1} . \quad S_{1}=S \cup\left\langle s_{1}\right\rangle \wedge S_{2}=S \cup S_{2}^{\prime} \wedge \\
& \forall s_{2} . s_{2} \in S_{2}^{\prime} \longrightarrow s_{1} \succ s_{2}
\end{aligned}
$$


- Let $\succ_{x}$ and $\succ_{y}$ be relations over $X$ and $Y$. Their lexicographic product, denoted by $\succ_{x}\langle * \operatorname{lex} *\rangle \succ_{y}$, is a relation $\succ$ on $X \times Y$ such that

$$
\left(x_{1}, y_{1}\right) \succ\left(x_{2}, y_{2}\right) \text { iff } x_{1} \succ_{x} x_{2} \vee\left(x_{1}=x_{2} \wedge y_{1} \succ_{y} y_{2}\right)
$$

- Let $\succ_{x}$ be a relation on $X$, and for each $x \in X$ let $\succ_{y}^{x}$ be a relation over $Y$ (i.e., let $\lambda x$. $\succ_{y}^{x}$ be a function mapping $X$ to relations on $Y$ ). Their parametrized lexicographic product 7 denoted by $\succ_{x}\left\langle * \operatorname{lex}^{\mathrm{p}} *\right\rangle \succ_{y}^{x}$, is a relation $\succ$ on $X \times Y$ such that

$$
\left(x_{1}, y_{1}\right) \succ\left(x_{2}, y_{2}\right) \text { iff } x_{1} \succ_{x} x_{2} \vee\left(x_{1}=x_{2} \wedge y_{1} \succ_{y}^{x_{1}} y_{2}\right) \text {. }
$$

Proposition 3.4 (Properties of well-founded relations).

- A relation $\succ$ is well-founded iff

$$
\forall Q .(\exists a \in Q) \longrightarrow\left(\exists a_{\text {min }} \in Q .\left(\forall a^{\prime} . a_{\text {min }} \succ a^{\prime} \longrightarrow a^{\prime} \notin Q\right)\right)
$$

- Let $\mathrm{f}$ be a function and $\succ$ a relation such that $x \succ y \longrightarrow \mathrm{f} x \succ^{\prime} \mathrm{f} y$. If $\succ^{\prime}$ is well-founded, then so is $\succ$.

- If $\succ$ is well-founded, then so is $\succ^{\text {mult }}$.

- Let $\succ_{x}$ be a well-founded relation on $X$ and for each $x \in X$ let be $\succ_{y}^{x}$ a well-founded relation. Then $\succ_{x}\left\langle * \operatorname{lex}^{\mathrm{p}} *\right\rangle \succ_{y}^{x}$ is well-founded.

\subsection{Logic of CNF formulae.}

Definition 3.5 (Basic types).

\begin{tabular}{ll}
\hline Variable & natural number \\
Literal & either a positive variable (Pos $v b l)$ or a negative variable (Neg $v b l)$ \\
Clause & a list of literals \\
Formula & a list of clauses \\
Valuation & a list of literals \\
Trail & a list of (Literal, bool) pairs \\
\hline
\end{tabular}

For the sake of readability, we will sometimes omit types and use the following naming convention: literals (i.e., variables of the type Literal) are denoted by $l$ (e.g., $l, l^{\prime}, l_{0}, l_{1}, l_{2}, \ldots$ ), variables by $v b l$, clauses by $c$, formulae by $F$, valuations by $v$, and trails by $M$.

Note that, in order to be closer to implementation (and to the standard solver input format - DIMACS), clauses and formulae are represented using lists instead of sets (a more detailed discussion on this issue is given in Section 9). Although a trail is not a list of literals (but rather a list of (Literal, bool) pairs), for simplicity, we will often identify it with its list of underlying literals, and we will treat trails as valuations. In addition, a trail can be implemented, not only as a list of (Literal, bool) pairs but in some other equivalent way. We abuse the notation and overload some symbols. For example, the symbol $\in$ denotes both set membership and list membership, and it is also used to denote that a literal occurs in a formula. Symbol vars is also overloaded and denotes the set of variables occurring in a clause, in a formula, or in a valuation.

Definition 3.6 (Literals and clauses related).

\footnotetext{
${ }^{7}$ Note that lexicographic product can be regarded as a special case of parametrized lexicographic product (where a same $\succ_{y}$ is used for each $x \in X$ ).
} 
- The opposite literal of a literal $l$, denoted by $\bar{l}$, is defined by: $\overline{\text { Pos } v b l}=\operatorname{Neg} v b l, \overline{\operatorname{Neg} v b l}=$ Pos vbl.

- A formula $F$ contains a literal $l$ (i.e., a literal $l$ occurs in a formula $F$ ), denoted by $l \in F$, iff $\exists$ c. $c \in F \wedge l \in c$.

- The set of variables that occur in a clause $c$ is denoted by vars $c$. The set of variables that occur in a formula $F$ is denoted by vars $F$. The set of variables that occur in a valuation $v$ is denoted by vars $v$.

- The resolvent of clauses $c_{1}$ and $c_{2}$ over the literal $l$, denoted resolvent $c_{1} c_{2} l$ is the clause $\left(c_{1} \backslash l\right) @\left(c_{2} \backslash \bar{l}\right)$.

- A clause $c$ is a tautological clause, denoted by clauseTautology $c$, if it contains both a literal and its opposite (i.e., $\exists l . l \in c \wedge \bar{l} \in c$ ).

- The conversion of a valuation $v$ to a formula is the list $\langle v\rangle$ that contains all single literal clauses made of literals from $v$.

Definition 3.7 (Semantics).

- A literal $l$ is true in a valuation $v$, denoted by $v \vDash l$, iff $l \in v$. A clause $c$ is true in a valuation $v$, denoted by $v \vDash c$, iff $\exists l . l \in c \wedge v \vDash l$. A formula $F$ is true in a valuation $v$, denoted by $v \vDash F$, iff $\forall c . c \in F \Rightarrow v \vDash c$.

- A literal $l$ is false in a valuation $v$, denoted by $v \vDash \neg l$, iff $\bar{l} \in v$. A clause $c$ is false in a valuation $v$, denoted by $v \vDash \neg c$, iff $\forall l . l \in c \Rightarrow v \vDash \neg l$. A formula $F$ is false in a valuation $v$, denoted by $v \vDash \neg F$, iff $\exists c . c \in F \wedge v \vDash \neg c$ 8

- $v \not \models l(v \not \models c / v \not \models F)$ denotes that $l(c / F)$ is not true in $v$ (then we say that $l(c / F)$ is unsatisfied in $v) . v \not \models \neg l(v \not \models \neg c / v \not \models \neg F)$ denotes that $l(c / F)$ is not false in $v$ (then we say that $l(c / F)$ is unfalsified in $v)$.

Definition 3.8 (Valuations and models).

- A valuation $v$ is inconsistent, denoted by inconsistent $v$, iff it contains both a literal and its opposite i.e., iff $\exists l . v \vDash l \wedge v \vDash \bar{l}$. A valuation is consistent, denoted by (consistent $v$ ), iff it is not inconsistent.

- A valuation $v$ is total with respect to a variable set $V b l$, denoted by total $v V b l$, iff vars $v \supseteq V b l$.

- A model of a formula $F$ is a consistent valuation under which $F$ is true. A formula $F$ is satisfiable, denoted by sat $F$, iff it has a model, i.e., $\exists v$. consistent $v \wedge v \vDash F$.

- A clause $c$ is unit in a valuation $v$ with a unit literal $l$, denoted by isUnit $c l v$ iff $l \in c$, $v \not \models l, v \not \models \neg l$ and $v \vDash \neg(c \backslash l)$ (i.e., $\forall l^{\prime} . l^{\prime} \in c \wedge l^{\prime} \neq l \Rightarrow v \vDash \neg l^{\prime}$ ).

- A clause $c$ is a reason for propagation of literal $l$ in valuation $v$, denoted by isReason $c l v$ iff $l \in c, v \vDash l, v \vDash \neg(c \backslash l)$, and for each literal $l^{\prime} \in(c \backslash l)$, the literal $\overline{l^{\prime}}$ precedes $l$ in $v$.

\footnotetext{
${ }^{8}$ Note that the symbol $\vDash \neg$ is atomic, i.e., $v \vDash \neg F$ does not correspond to $v \models(\neg F)$, although it would be the case if all propositional formulae (instead of CNF only) were considered.
} 
Definition 3.9 (Entailment and logical equivalence).

- A formula $F$ entails a clause $c$, denoted by $F \vDash c$, iff $c$ is true in every model of $F$. A formula $F$ entails a literal $l$, denoted by $F \vDash l$, iff $l$ is true in every model of $F$. A formula $F$ entails valuation $v$, denoted by $F \vDash v$, iff it entails all its literals i.e., $\forall l . l \in v \Rightarrow F \vDash l$. A formula $F_{1}$ entails a formula $F_{2}$, denoted by $F_{1} \vDash F_{2}$, if every model of $F_{1}$ is a model of $F_{2}$.

- Formulae $F_{1}$ and $F_{2}$ are logically equivalent, denoted by $F_{1} \equiv F_{2}$, iff any model of $F_{1}$ is a model of $F_{2}$ and vice versa, i.e., iff $F_{1} \vDash F_{2}$ and $F_{2} \vDash F_{1}$.

Definition 3.10 (Trails related).

- For a trail element $a$, element $a$ denotes the first (Literal) component and isDecision $a$ denotes the second (Boolean) component. For a trail $M$, elements $M$ denotes the list of all its elements and decisions $M$ denotes the list of all its marked elements (i.e., of all its decision literals).

- The last decision literal, denoted by lastDecision $M$, is the last marked element of the list $M$, i.e., lastDecision $M=$ last (decisions $M$ ).

- decisionsTo $M l$ is the list of all marked elements from a trail $M$ that precede the first occurrence of the element $l$, including $l$ if it is marked, i.e., decisionsTo $l M=$ decisions (prefixTo $l M$ ).

- The current level for a trail $M$, denoted by currentLevel $M$, is the number of marked literals in $M$, i.e., currentLevel $M=$ length (decisions $M$ ).

- The decision level of a literal $l$ in a trail $M$, denoted by level $l M$, is the number of marked literals in the trail that precede the first occurrence of $l$, including $l$ if it is marked, i.e., level $l M=$ length (decisionsTo $M l$ ).

- prefixToLevel $M$ level is the prefix of a trail $M$ containing all elements of $M$ with levels less or equal to level.

- The prefix before last decision, denoted by prefixBeforeLastDecision $M$, is a prefix of the trail $M$ before its last marked element (not including it).9

- The last asserted literal of a clause $c$, denoted by lastAssertedLiteral $c M$, is the literal from $c$ that is in $M$, such that no other literal from $c$ comes after it in $M$.

- The maximal level of a literal in the clause $c$ with respect to a trail $M$, denoted by maxLevel $c M$, is the maximum of all levels of literals from $c$ asserted in $M$.

Example 3.11. A trail $M$ could be $\left[+1^{i},-2^{d},+6^{i},+5^{d},-3^{i},+4^{i},-7^{d}\right]$. The symbol + is written instead of the constructor Pos, the symbol - instead of Neg. decisions $M=$ $\left[-2^{d},+5^{d},-7^{d}\right]$, lastDecision $M=-7$, decisionsTo $M+4=\left[-2^{d},+5^{d}\right]$, and decisionsTo $M-7$ $=\left[-2^{d},+5^{d},-7^{d}\right]$. level $+1 M=0$, level $+4 M=2$, level $-7 M=3$, currentLevel $M=3$, prefixToLevel $M 1=\left[+1^{i},+2^{d},+6^{i}\right]$. If $c$ is $[+4,+6,-3]$, then lastAssertedLiteral $c M=+4$, and maxLevel $c M=2$.

\footnotetext{
${ }^{9}$ Note that some of these functions are used only for some trails. For example, prefixBeforeLastDecision $M$ makes sense only for trails that contain at least one decision literal. Nevertheless, these functions are still defined as total functions - for example, prefixBeforeLastDecision $M$ equals $M$ if there are no decision literals.
} 


\section{DPLL SEARCH}

In this section we consider a basic transition system that contains only transition rules corresponding to steps used in the original DPLL procedure: unit propagation, backtracking, and making decisions for branching (described informally in Section 2.1) the pure literal step is usually not used within modern SAT solvers, so it will be omitted). These rules will be defined in the form of relations over states, in terms of the logic described in Section 3. It will be proved that the system containing these rules is terminating, sound and complete. The rules within the system are not ordered and the system is sound, terminating, and complete regardless of any specific ordering. However, it will be obvious that better performance is obtained if making decisions is maximally postponed, in the hope that it will not be necessary.

4.1. States and Rules. The state of the solver performing the basic DPLL search consists of the formula $F$ being tested for satisfiability (that remains unchanged) and the trail $M$ (that may change during the solver's operation). The only parameter to the solver is the set of variables DecVars used for branching. By Vars we will denote the set of all variables encountered during solving - these are the variables from the initial formula $F_{0}$ and the decision variables DecVars, i.e., Vars $=$ vars $F_{0} \cup$ DecVars.

Definition 4.1 (State). A state of the system is a pair $(M, F)$, where $M$ is a trail and $F$ is a formula. A state $\left([], F_{0}\right)$ is an initial state for the input formula $F_{0}$.

Transition rules are introduced by the following definition, in the form of relations over states.

Definition 4.2 (Transition rules).

$$
\begin{aligned}
& \text { unitPropagate }\left(M_{1}, F_{1}\right)\left(M_{2}, F_{2}\right) \quad \text { iff } \quad \exists c l . \quad c \in F_{1} \wedge \text { isUnit } c l M_{1} \wedge \\
& M_{2}=M_{1} @ l^{i} \wedge F_{2}=F_{1} \\
& \text { backtrack }\left(M_{1}, F_{1}\right)\left(M_{2}, F_{2}\right) \text { iff } M_{1} \vDash \neg F_{1} \wedge \text { decisions } M_{1} \neq[] \wedge \\
& M_{2}=\text { prefixBeforeLastDecision } M_{1} @{\overline{\text { lastDecision } M_{1}}}^{i} \wedge F_{2}=F_{1} \\
& \text { decide }\left(M_{1}, F_{1}\right)\left(M_{2}, F_{2}\right) \quad \text { iff } \quad \exists l . \quad \operatorname{var} l \in \text { DecVars } \wedge l \notin M_{1} \wedge \bar{l} \notin M_{1} \wedge \\
& M_{2}=M_{1} @ l^{d} \wedge F_{2}=F_{1}
\end{aligned}
$$

As can be seen from the above definition (and in accordance with the description given in Section 2.1), the rule unitPropagate uses a unit clause - a clause with only one literal $l$ undefined in $M_{1}$ and with all other literals false in $M_{1}$. Such a clause can be true only if $l$ is true, so this rule extends $M_{1}$ by $l$ (as an implied literal). The rule backtrack is applied when $F_{1}$ is false in $M_{1}$. Then it is said that a conflict occurred, and clauses from $F_{1}$ that are false in $M_{1}$ are called conflict clauses. In that case, the last decision literal $l^{d}$ in $M_{1}$ and all literals that succeed it are removed from $M_{1}$, and the obtained prefix is extended by $\bar{l}^{i}$ as an implied literal. The rule decide extends the trail by an arbitrary literal $l$ as a decision literal, such that the variable of $l$ belongs to DecVars and neither $l$ nor $\bar{l}$ occur in $M_{1}$. In that case, we say there is a branching on $l$.

The transition system considered is described by the relation $\rightarrow_{d}$, introduced by the following definition. 
Definition $4.3\left(\rightarrow_{d}\right)$.

$s_{1} \rightarrow_{d} s_{2}$ iff unitPropagate $s_{1} s_{2} \vee$ backtrack $s_{1} s_{2} \vee$ decide $s_{1} s_{2}$

Definition 4.4 (Outcome states). An outcome state is either an accepting state or a rejecting state.

A state is an accepting state if $M \not \models \neg F$ and there is no state $\left(M^{\prime}, F^{\prime}\right)$ such that decide $(M, F)\left(M^{\prime}, F^{\prime}\right)$ (i.e., there is no literal such that var $l \in \operatorname{DecVars,} l \notin M$, and $\bar{l} \notin M)$.

A state is a rejecting state if $M \vDash \neg F$ and decisions $M=[]$.

Note that the condition $M \not \models \neg F$ in the above definition can be replaced by the condition $M \models F$, but the former is used since its check can be more efficiently implemented.

Example 4.5. Let $F_{0}=[[-1,+2],[-1,-3,+5,+7],[-1,-2,+5,-7],[-2,+3],[+2,+4]$, $[-2,-5,+7],[-3,-6,-7],[-5,+6]]$. One possible $\rightarrow_{d}$ trace is given below.

\begin{tabular}{|c|c|}
\hline & \\
\hline decide $(l=+1)$ & {$\left[+1^{d}\right]$} \\
\hline unitPropagate $(c=[-1,+2], l=+2)$ & {$\left[+1^{d},+2^{i}\right]$} \\
\hline unitPropagate $(c=[-2,+3], l=+3)$ & {$\left[+1^{d},+2^{i},+3^{i}\right]$} \\
\hline decide $(l=+4)$ & {$\left[+1^{d},+2^{i},+3^{i},+4^{d}\right]$} \\
\hline decide $(l=+5)$ & {$\left[+1^{d},+2^{i},+3^{i},+4^{d},+5^{d}\right]$} \\
\hline unitPropagate $(c=[-5,+6], l=+6)$ & {$\left[+1^{d},+2^{i},+3^{i},+4^{d},+5^{d},+6^{i}\right]$} \\
\hline unitPropagate $(c=[-2,-5,+7], l=+7)$ & {$\left[+1^{d},+2^{i},+3^{i},+4^{d},+5^{d},+6^{i},+7^{i}\right]$} \\
\hline $\operatorname{backtrack}(M \models \neg[-3,-6,-7])$ & {$\left[+1^{d},+2^{i},+3^{i},+4^{d},-5^{i}\right]$} \\
\hline unitPropagate $(c=[-1,-3,+5,+7], l=+7)$ & {$\left[+1^{d},+2^{i},+3^{i},+4^{d},-5^{i},+7^{i}\right]$} \\
\hline $\operatorname{backtrack}(M \vDash \neg[-1,-2,+5,-7])$ & {$\left[+1^{d},+2^{i},+3^{i},-4^{i}\right]$} \\
\hline decide $(l=+5)$ & {$\left[+1^{d},+2^{i},+3^{i},-4^{i},+5^{d}\right]$} \\
\hline unitPropagate $(c=[-5,+6], l=+6)$ & {$\left[+1^{d},+2^{i},+3^{i},-4^{i},+5^{d},+6^{i}\right]$} \\
\hline unitPropagate $(c=[-2,-5,+7], l=+7)$ & {$\left[+1^{d},+2^{i},+3^{i},-4^{i},+5^{d},+6^{i},+7^{i}\right]$} \\
\hline $\operatorname{backtrack}(M \vDash \neg[-3,-6,-7])$ & {$\left[+1^{d},+2^{i},+3^{i},-4^{i},-5^{i}\right]$} \\
\hline unitPropagate $(c=[-1,-3,+5,+7], l=+$ & {$\left[+1^{d},+2^{i},+3^{i},-4^{i},-5^{i},+7^{i}\right]$} \\
\hline backtrack $(M \vDash \neg[-1,-2,+5,-7]$ & {$\left[-1^{i}\right]$} \\
\hline decide $(l=+2)$ & {$\left[-1^{i},+2^{d}\right]$} \\
\hline unitPropagate $(c=[-2,+3], l=+3)$ & {$\left[-1^{i},+2^{d},+3^{i}\right]$} \\
\hline decide $(l=$ & {$\left[-1^{i},+2^{d},+3^{i},+4^{d}\right]$} \\
\hline decide $(l=+5)$ & {$\left[-1^{i},+2^{d},+3^{i},+4^{d},+5^{d}\right]$} \\
\hline Dagate $(c=[-5,+6], l=+6)$ & {$\left[-1^{i},+2^{d},+3^{i},+4^{d},+5^{d},+6^{i}\right]$} \\
\hline unitPropagate $(c=[-2,-5,+7], l=+7)$ & {$\left[-1^{i},+2^{d},+3^{i},+4^{d},+5^{d},+6^{i},+7^{i}\right]$} \\
\hline backtrack $M \vDash \neg[-3,-6,-7]$ & {$\left[-1^{i},+2^{d},+3^{i},+4^{d},-5^{i}\right]$} \\
\hline decide $(l=+6)$ & {$\left[-1^{i},+2^{d},+3^{i},+4^{d},-5^{i},+6^{d}\right]$} \\
\hline unitPropagate $(c=[-3,-6,-7], l=-7)$ & {$\left[-1^{i},+2^{d},+3^{i},+4^{d},-5^{i},+6^{d},-7^{i}\right]$} \\
\hline
\end{tabular}

4.2. Properties. In order to prove that the presented transition system is terminating, sound, and complete, first, local properties of the transition rules have to be given in the form of certain invariants. 
4.2.1. Invariants. For proving properties of the described transition system, several relevant rule invariants will be used (not all of them are used for proving each of soundness, completeness, and termination, but we list them all here for the sake of simplicity).

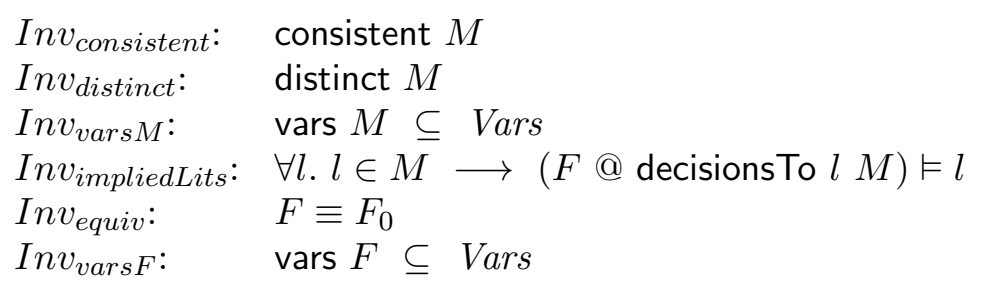

The condition Inv $v_{\text {consistent }}$ states that the trail $M$ can potentially be a model of the formula, and Inv distinct requires that it contains no repeating elements. The Inv $v_{\text {impliedLits }}$ ensures that any literal $l$ in $M$ is entailed by $F$ with all decision literals that precede $l$.

Notice that the given rules do not change formulae in states, so it trivially holds that $F=F_{0}$, which further implies Invequiv and Inv varsF. However, the transition systems that follow in the next sections may change formulae, so the above set of invariants is more appropriate. If only testing satisfiability is considered (and not in building models for satisfiable formulae), instead of $I n v_{\text {equiv }}$, it is sufficient to require that $F$ and $F_{0}$ are weakly equivalent (i.e., equisatisfiable).

The above conditions are indeed invariants (i.e., they are met for each state during the application of the rules), as stated by the following lemma.

\section{Lemma 4.6.}

(1) In the initial state $\left([], F_{0}\right)$ all the invariants hold.

(2) If $(M, F) \rightarrow_{d}\left(M^{\prime}, F^{\prime}\right)$ and if the invariants are met in the state $(M, F)$, then they are met in the state $\left(M^{\prime}, F^{\prime}\right)$ too.

(3) If $\left([], F_{0}\right) \rightarrow_{d}^{*}(M, F)$, then all the invariants hold in the state $(M, F)$.

The proof of this lemma considers a number of cases — one for each rule-invariant pair.

4.2.2. Soundness. Soundness of the given transition system requires that if the system terminates in an accepting state, then the input formula is satisfiable, and if the system terminates in a rejecting state, then the input formula is unsatisfiable.

The following lemma ensures soundness for satisfiable input formulae, and the next one is used for proving soundness for unsatisfiable input formulae (but also in some other contexts).

Lemma 4.7. If DecVars $\supseteq$ vars $F_{0}$ and if there is an accepting state $(M, F)$ such that:

(1) consistent $M$ (i.e., Inv consistent holds),

(2) $F \equiv F_{0}$ (i.e., Inv equiv holds),

(3) vars $F \subseteq$ Vars (i.e., Inv varsF holds),

then the formula $F_{0}$ is satisfiable and $M$ is one model (i.e., model $M F_{0}$ ).

Lemma 4.8. If there is a state $(M, F)$ such that:

(1) $\forall l . l \in M \longrightarrow(F$ @ decisionsTo $l M) \vDash l$ (i.e., InvimpliedLits holds),

(2) $M \vDash \neg F$

then $\neg($ sat $(F @$ decisions $M))$.

Theorem 4.9 (Soundness for $\rightarrow_{d}$ ). If $\left([], F_{0}\right) \rightarrow_{d}^{*}(M, F)$, then: 
(1) If DecVars $\supseteq$ vars $F_{0}$ and $(M, F)$ is an accepting state, then the formula $F_{0}$ satisfiable and $M$ is one model (i.e., sat $F_{0}$ and model $M F_{0}$ ).

(2) If $(M, F)$ is a rejecting state, then the formula $F_{0}$ is unsatisfiable (i.e., $\neg\left(\right.$ sat $\left.F_{0}\right)$ ).

Proof. By Lemma 4.6 all the invariants hold in the state $(M, F)$.

Let us assume that DecVars $\supseteq$ vars $F_{0}$ and $(M, F)$ is an accepting state. Then, by Lemma 4.7, the formula is $F_{0}$ satisfiable and $M$ is one model.

Let us assume that $(M, F)$ is a rejecting state. Then $M \vDash \neg F$ and, by Lemma 4.8, $\neg$ (sat $(F @($ decisions $M)))$. Since $(M, F)$ is a rejecting state, it holds that decisions $M=[]$, and hence $\neg($ sat $F)$. From $F \equiv F_{0}\left(\right.$ Inv $\left._{\text {equiv }}\right)$, it follows that $\neg\left(\right.$ sat $\left.F_{0}\right)$, i.e., the formula $F_{0}$ is unsatisfiable.

4.2.3. Termination. Full and precise formalization of termination is very demanding, and termination proofs given in the literature (e.g., [KG07, NOT06]) are far from detailed formal proofs. For this reason, termination proofs will be presented here in more details, including auxiliary lemmas used to prove the termination theorem.

The described transition system terminates, i.e., for any input formulae $F_{0}$, the system (starting from the initial state $\left([], F_{0}\right)$ ) will reach a final state in a finite number of steps. In other words, the relation $\rightarrow_{d}$ is well-founded. This can be proved by constructing a wellfounded partial ordering $\succ$ over trails, such that $\left(M_{1}, F_{1}\right) \rightarrow_{d}\left(M_{2}, F_{2}\right)$ implies $M_{1} \succ M_{2}$. In order to reach this goal, several auxiliary orderings are defined.

First, a partial ordering over annotated literals $\succ_{\text {lit }}$ and a partial ordering over trails $\succ_{\text {tr }}$ will be introduced and some of their properties will be given within the following lemmas.

Definition $4.10\left(\succ_{\text {lit }}\right) \cdot l_{1} \succ_{\text {lit }} l_{2}$ iff isDecision $l_{1} \wedge \neg\left(\right.$ isDecision $\left.l_{2}\right)$

Lemma 4.11. $\succ_{\text {lit }}$ is transitive and irreflexive.

Definition $4.12\left(\succ_{\text {tr }}\right) \cdot M_{1} \succ_{\text {tr }} M_{2}$ iff $M_{1} \succ_{\text {lit }}^{\text {lex }} M_{2}$, where $\succ_{\text {lit }}^{\text {lex }}$ is a lexicographic extension of $\succ_{\text {lit. }}$.

Lemma 4.13. $\succ_{\mathrm{tr}}$ is transitive, irreflexive, and acyclic (i.e., there is no trail $M$ such that $\left.M \succ_{\operatorname{tr}}^{+} M\right)$.

For any three trails $M, M^{\prime}$, and $M^{\prime \prime}$ it holds that: if $M^{\prime} \succ_{\operatorname{tr}} M^{\prime \prime}$, then $M @ M^{\prime} \succ_{\operatorname{tr}}$ $M @ M^{\prime \prime}$.

The next lemma links relations $\rightarrow_{d}$ and $\succ_{\mathrm{tr}}$.

Lemma 4.14. If decide $\left(M_{1}, F_{1}\right)\left(M_{2}, F_{2}\right)$ or unitPropagate $\left(M_{1}, F_{1}\right)\left(M_{2}, F_{2}\right)$ or backtrack $\left(M_{1}, F_{1}\right)\left(M_{2}, F_{2}\right)$, then $M_{1} \succ_{\text {tr }} M_{2}$.

The relation $\succ_{\operatorname{tr}}$ is not necessarily well-founded (for the elements of the trails range over infinite sets), so a restriction $\left.\succ_{\operatorname{tr}}\right|_{V b l}$ of the relation $\succ_{\text {tr }}$ will be defined such that it is well-founded, which will lead to the termination proof for the system.

Definition $\left.4.15\left(\left.\succ_{\mathrm{tr}}\right|_{V b l}\right) \cdot M_{1} \succ_{\mathrm{tr}}\right|_{V b l} M_{2}$ iff $\left(\right.$ distinct $M_{1} \wedge$ vars $\left.M_{1} \subseteq V b l\right) \wedge\left(\right.$ distinct $M_{2} \wedge$ vars $\left.M_{2} \subseteq V b l\right) \wedge M_{1} \succ_{\text {tr }} M_{2}$

Lemma 4.16. If the set $V b l$ is finite, then the relation $\left.\succ_{\mathrm{tr}}\right|_{V b l}$ is a well-founded ordering.

Finally, we prove that the transition system is terminating. 
Theorem 4.17 (Termination for $\rightarrow_{d}$ ). If the set DecVars is finite, for any formula $F_{0}$, the relation $\rightarrow_{d}$ is well-founded on the set of states $(M, F)$ such that $\left([], F_{0}\right) \rightarrow_{d}^{*}(M, F)$.

Proof. By Proposition 3.4 it suffices to construct a well-founded ordering on the set of states $(M, F)$ such that $\left([], F_{0}\right) \rightarrow_{d}^{*}(M, F)$ such that

$$
\left(M_{1}, F_{1}\right) \rightarrow_{d}\left(M_{2}, F_{2}\right) \longrightarrow\left(M_{1}, F_{1}\right) \succ\left(M_{2}, F_{2}\right) .
$$

One such ordering is $\succ$ defined by: $\left(M_{1}, F_{1}\right) \succ\left(M_{2}, F_{2}\right)$ iff $\left.M_{1} \succ_{\mathrm{tr}}\right|_{\text {Vars }} M_{2}$.

Indeed, since by Lemma 4.16, $\left.\succ_{\text {tr }}\right|_{\text {Vars }}$ is well-founded, by Proposition 3.4 (for a function mapping $(M, F)$ to $M), \succ$ is also a well-founded ordering.

Let $\left(M_{1}, F_{1}\right)$ and $\left(M_{2}, F_{2}\right)$ be two states such that $\left([], F_{0}\right) \rightarrow_{d}^{*}\left(M_{1}, F_{1}\right)$ and $\left(M_{1}, F_{1}\right) \rightarrow_{d}$ $\left(M_{2}, F_{2}\right)$. By Lemma 4.6 all the invariants hold for $\left(M_{1}, F_{1}\right)$. From $\left(M_{1}, F_{1}\right) \rightarrow_{d}\left(M_{2}, F_{2}\right)$, by Lemma 4.14, it follows that $M_{1} \succ_{\operatorname{tr}} M_{2}$. Moreover, by Lemma 4.6, all the invariants hold also for $\left(M_{2}, F_{2}\right)$, so distinct $M_{1}$, vars $M_{1} \subseteq$ Vars, distinct $M_{2}$ and vars $M_{2} \subseteq$ Vars. Ultimately, $M_{1} \succ_{\text {tr }} \mid$ Vars $M_{2}$.

4.2.4. Completeness. Completeness requires that all final states are outcome states.

Theorem 4.18 (Completeness for $\rightarrow_{d}$ ). Each final state is either accepting or rejecting.

Proof. Let $(M, F)$ be a final state. It holds that either $M \vDash \neg F$ or $M \not \models \neg F$.

If $M \not \models \neg F$, since there is no state $\left(M^{\prime}, F^{\prime}\right)$ such that decide $(M, F)\left(M^{\prime}, F^{\prime}\right)$ (as $(M, F)$ is a final state), there is no literal $l$ such that var $l \in \operatorname{Dec} \operatorname{Vars}, l \notin M$, and $\bar{l} \notin M$, so $(M, F)$ is an accepting state.

If $M \vDash \neg F$, since there is no state $\left(M^{\prime}, F^{\prime}\right)$ such that backtrack $(M, F)\left(M^{\prime}, F^{\prime}\right)$ (as $(M, F)$ is a final state), it holds that decisions $M=[]$, so $(M, F)$ is a rejecting state.

Notice that from the proof it is clear that the basic search system consisting only of the rules decide and backtrack is complete.

4.2.5. Correctness. The theorems 4.9, 4.17, and 4.18 directly lead to the theorem about correctness of the introduced transition system 10

Theorem 4.19 (Correctness for $\rightarrow_{d}$ ). The given transition system is correct, i.e., if all variables of the input formula belong to the set DecVars, then for any satisfiable input formula, the system terminates in an accepting state, and for any unsatisfiable formula, the system terminates in a rejecting state.

\section{BACKJUMPING}

In this section, we consider a transition system that replaces naive chronological backtracking by more advanced nonchronological backjumping.

\footnotetext{
${ }^{10}$ Correctness of the system can be proved with a weaker condition. Namely, instead of the condition that all variables of the input formula belong to the set DecVars, it is sufficient that all strong backdoor variables belong to DecVars [BHMW09, but that weaker condition is not considered here.
} 
5.1. States and Rules. The rules of the new system are given (as in Section 4) in the form of relations over states.

Definition 5.1 (Transition rules). unitPropagate $\left(M_{1}, F_{1}\right)\left(M_{2}, F_{2}\right)$ iff

$$
\begin{array}{ll}
\exists c l . & F_{1} \vDash c \wedge \operatorname{var} l \in \text { Vars } \wedge \text { isUnit } c l M_{1} \wedge \\
& M_{2}=M_{1} @ l^{i} \wedge F_{2}=F_{1}
\end{array}
$$

$$
\begin{aligned}
& \text { backjump }\left(M_{1}, F_{1}\right)\left(M_{2}, F_{2}\right) \text { iff } \\
& \exists c l P \text { level. } \quad F_{1} \vDash c \wedge \operatorname{var} l \in \text { Vars } \wedge \\
& P=\text { prefixToLevel level } M \wedge 0 \leq \text { level }<\text { currentLevel } M \wedge \\
& \text { isUnit } \operatorname{cl} P \wedge \\
& F_{2}=F_{1} \wedge M_{2}=P @ l^{i} \\
& \text { decide }\left(M_{1}, F_{1}\right)\left(M_{2}, F_{2}\right) \quad \text { iff } \quad \exists l . \quad \operatorname{var} l \in \text { DecVars } \wedge l \notin M_{1} \wedge \bar{l} \notin M_{1} \wedge \\
& M_{2}=M_{1} @ l^{d} \wedge F_{2}=F_{1}
\end{aligned}
$$

In the following, the transition system described by the relation $\rightarrow_{b}$ defined by these rules will be considered.

The key difference between the new transition system and one built over the rules given in Definition 4.2 is the rule backjump (that replaces the rule backtrack). The rule decide is the same as the one given in Definition 4.2, while the rule unitPropagate is slightly modified (i.e., its guard is relaxed).

The clause $c$ in the backjump rule is called a backjump clause and the level level is called a backjump level. The given definition of the backjump rule is very general - it does not specify how the backjump clause $c$ is constructed and what prefix $P$ (i.e., the level level) is chosen if there are several options. There are different strategies that specify these choices and they are required for concrete implementations. The conditions that $P$ is a prefix to a level (i.e., that $P$ is followed by a decision literal in $M_{1}$ ) and that this level is smaller than the current level are important only for termination. Soundness can be proved even with a weaker assumption that $P$ is an arbitrary prefix of $M_{1}$. However, usually the shortest possible prefix $P$ is taken. The backtrack rule can be seen as a special case of the backjump rule. In that special case, the clause $c$ is built of opposites of all decision literals in the trail and $P$ becomes prefixBeforeLastDecision $M_{1}$.

Notice that the backjump clause $c$ does not necessarily belong to $F_{1}$ but can be an arbitrary logical consequence of it. So, instead of $c \in F_{1}$, weaker conditions $F_{1} \vDash c$ and var $l \in$ Vars are used in the backjump rule (the latter condition is important only for termination). This weaker condition (inspired by the use of SAT engines in SMT solvers) can be used also for the unitPropagate rule and leads from the rule given in Definition 4.2, to its present version (this change is not relevant for the system correctness). The new version of unitPropagate has much similarities with the backjump rule - the only difference is that the backjump rule always asserts the implied literal to a proper prefix of the trail.

Example 5.2. Let $F_{0}$ be the same formula as in Example 4.5. One possible $\rightarrow_{b}$ trace is given below. Note that, unlike in the trace shown in Example 4.5, the decision literal +4 is removed from the trail during backjumping, since it was detected to be irrelevant for the conflict, resulting in a shorter trace. The deduction of backjump clauses (e.g., $[-2,-3,-5]$ ) will be presented in Example 7.4 . 


\begin{tabular}{|c|c|}
\hline rule & \\
\hline $\begin{array}{l}\text { decide }(l=+1) \\
\text { unitPropagate }(c=[-1,+2], l=+2) \\
\text { unitPropagate }(c=[-2,+3], l=+3) \\
\text { decide }(l=+4) \\
\text { decide }(l=+5) \\
\text { unitPropagate }(c=[-5,+6], l=+6) \\
\text { unitPropagate }(c=[-2,-5,+7], l=+7) \\
\text { backjump }(c=[-2,-3,-5], l=-5) \\
\text { unitPropagate }(c=[-1,-3,+5,+7], l=+7) \\
\text { backjump }(c=[-1], l=-1) \\
\text { decide }(l=+2) \\
\text { unitPropagate }(c=[-2,+3], l=+3) \\
\text { decide }(l=+4) \\
\quad \text { decide }(l=+5) \\
\text { unitPropagate }(c=[-5,+6], l=+6) \\
\text { unitPropagate }(c=[-2,-5,+7], l=+7) \\
\text { backjump }(c=[-2,-3,-5]) \\
\text { decide }(l=+4) \\
\text { decide }(l=+6) \\
\text { unitPropagate }(c=[-3,-6,-7], l=-7)\end{array}$ & $\begin{array}{l}{\left[+1^{d}\right]} \\
{\left[+1^{d},+2^{i}\right]} \\
{\left[+1^{d},+2^{i},+3^{i}\right]} \\
{\left[+1^{d},+2^{i},+3^{i},+4^{d}\right]} \\
{\left[+1^{d},+2^{i},+3^{i},+4^{d},+5^{d}\right]} \\
{\left[+1^{d},+2^{i},+3^{i},+4^{d},+5^{d},+6^{i}\right]} \\
{\left[+1^{d},+2^{i},+3^{i},+4^{d},+5^{d},+6^{i},+7^{i}\right]} \\
{\left[+1^{d},+2^{i},+3^{i},-5^{i}\right]} \\
{\left[+1^{d},+2^{i},+3^{i},-5^{i},+7^{i}\right]} \\
{\left[-1^{i}\right]} \\
{\left[-1^{i},+2^{d}\right]} \\
{\left[-1^{i},+2^{d},+3^{i}\right]} \\
{\left[-1^{i},+2^{d},+3^{i},+4^{d}\right]} \\
{\left[-1^{i},+2^{d},+3^{i},+4^{d},+5^{d}\right]} \\
{\left[-1^{i},+2^{d},+3^{i},+4^{d},+5^{d},+6^{i}\right]} \\
{\left[-1^{i},+2^{d},+3^{i},+4^{d},+5^{d},+6^{i},+7^{i}\right]} \\
{\left[-1^{i},+2^{d},+3^{i},-5^{i}\right]} \\
{\left[-1^{i},+2^{d},+3^{i},-5^{i},+4^{d}\right]} \\
{\left[-1^{i},+2^{d},+3^{i},-5^{i},+4^{d},+6^{d}\right]} \\
{\left[-1^{i},+2^{d},+3^{i},-5^{i},+4^{d},+6^{d},-7^{i}\right]}\end{array}$ \\
\hline
\end{tabular}

5.2. Backjump Levels. In Definition 5.1, for the backjump rule to be applicable, it is required that there is a level of the trail such that the backjump clause is unit in the prefix to that level. The following definition gives a stronger condition (used in modern SAT solvers) for a level ensuring applicability of the backjump rule to that level.

Definition 5.3 (Backjump level). A backjump level for the given backjump clause $c$ (false in $M$ ) is a level level that is strictly less than the level of the last falsified literal from $c$, and greater or equal to the levels of the remaining literals from $c$ :

$$
\begin{aligned}
\text { isBackjumpLevel level } l \text { c } M \quad \text { iff } \quad & M \vDash \neg c \wedge \bar{l}=\text { lastAssertedLiteral } \bar{c} M \wedge \\
& 0 \leq \text { level }<\text { level } \bar{l} M \wedge \\
& \forall l^{\prime} . l^{\prime} \in c \backslash l \longrightarrow \text { level } \overline{l^{\prime}} M \leq \text { level }
\end{aligned}
$$

Using this definition, the backjump rule can be defined in a more concrete and more operational way.

$$
\begin{aligned}
\text { backjump' }^{\prime}\left(M_{1}, F_{1}\right)\left(M_{2}, F_{2}\right) \quad \text { iff } \quad & \exists \text { cl level. } F_{1} \vDash c \wedge \text { var } l \in \text { Vars } \wedge \\
& \text { isBackjumpLevel level } l c M_{1} \wedge \\
& M_{2}=\left(\text { prefixToLevel level } M_{1}\right) @ l^{i} \wedge F_{2}=F_{1}
\end{aligned}
$$

Notice that, unlike in Definition [5.1, it is required that the backjump clause is false, so this new rule is applicable only in conflict situations.

It still remains unspecified how the clause $c$ is constructed. Also, it is required to check whether the clause $c$ is false in the current trail $M$ and implied by the current formula $F$. In 
Section 7 it will be shown that if a clause $c$ is built during a conflict analysis process, these conditions will hold by construction and so it will not be necessary to check them explicitly. Calculating the level of each literal from $c$ (required for the backjump level condition) will also be avoided.

The following lemmas connect the backjump and backjump' rules.

Lemma 5.4. If:

(1) consistent $M$ (i.e., Inv consistent holds),

(2) unique $M$ (i.e., Inv unique holds),

(3) isBackjumpLevel level $l$ c $M$,

then isUnit $c l$ (prefixToLevel level $M$ ).

Lemma 5.5. If a state $(M, F)$ satisfies the invariants and if backjump $(M, F)\left(M^{\prime}, F^{\prime}\right)$, then backjump $(M, F)\left(M^{\prime}, F^{\prime}\right)$.

Because of the very close connection between the relations backjump and backjump', we will not explicitly define two different transition relations $\rightarrow_{b}$. Most of the correctness arguments apply to both these relations, and hence only differences will be emphasized.

Although there are typically many levels satisfying the backjump level condition, (i.e., backjumping can be applied for each level between the level of the last falsified literal from $c$ and the levels of the remaining literals from $c$ ), usually it is applied to the lowest possible level, i.e., to the level that is a backjump level such that there is no smaller level that is also a backjump level. The following definition introduces formally the notion of a minimal backjump level.

Definition 5.6 (isMinimalBackjumpLevel). isMinimalBackjumpLevel level $l$ c $M$ iff isBackjumpLevel level l c $M \wedge\left(\forall\right.$ level $^{\prime}<$ level. $\neg$ isBackjumpLevel level ${ }^{\prime}$ l c $\left.M\right)$

Although most solvers use minimal levels when backjumping, this will be formally required only for systems introduced in Section 8 .

5.3. Properties. As in Section 4, local properties of the transition rules in the form of certain invariants are used in proving properties of the transition system.

5.3.1. Invariants. The invariants required for proving soundness, termination, and completeness of the new system are the same as the invariants listed in Section 4. So, it is required to prove that the rules backjump and the modified unitPropagate preserve all the invariants. Therefore, Lemma 4.6 has to be updated to address new rules and its proof has to be modified to reflect the changes in the definition of the transition relation.

5.3.2. Soundness and Termination. The soundness theorem (Theorem 4.9) has to be updated to address the new rules, but its proof remains analogous to the one given in Section 4.

The termination theorem (Theorem 4.17) also has to be updated, and its proof again remains analogous to the one given in 4. However, in addition to Lemma 4.14, the following lemma has to be used.

Lemma 5.7. If backjump $\left(M_{1}, F_{1}\right)\left(M_{2}, F_{2}\right)$, then $M_{1} \succ_{\operatorname{tr}} M_{2}$. 
This proof relies on the following property of the relation $\succ_{\mathrm{tr}}$.

Lemma 5.8. If $M$ is a trail and $P=$ prefixToLevel level $M$, such that $0 \leq$ level $<$ currentLevel $M$, then $M \succ_{\operatorname{tr}} P @ l^{i}$.

5.3.3. Completeness and Correctness. Completeness of the system is proved partly in analogy with the completeness proof of the system described in Section 4 , given in Theorem 4.18. When $(M, F)$ is a final state and $M \not \models \neg F$, the proof remains the same as for Theorem 4.18. When $(M, F)$ is a final state and $M \vDash \neg F$, for the new system it is not trivial that this state is a rejecting state (i.e., it is not trivial that decisions $M=[]$ ). Therefore, it has to be proved, given that the invariants hold, that if backjumping is not applicable in a conflict situation (when $M \vDash \neg F$ ), then decisions $M=$ [] (i.e., if decisions $M \neq$ [], then backjump' is applicable, and so is backjump). The proof relies on the fact that a backjump clause may be constructed only of all decision literals. This is the simplest way to construct a backjump clause $c$ and in this case backjumping degenerates to backtracking. The clause $c$ constructed in this way meets sufficient (but, of course, not necessary) conditions for the applicability of backjump' (and, consequently, by Lemma 5.5, for the applicability of backjump).

Lemma 5.9. If for a state $(M, F)$ it holds that:

(1) consistent $M$ (i.e., Inv consistent holds),

(2) unique $M$ (i.e., Inv unique holds),

(3) $\forall l . l \in M \longrightarrow F @$ (decisionsTo $l M) \vDash l$ (i.e., Inv impliedLits holds),

(4) vars $M \subseteq$ Vars (i.e., Inv vars $M$ holds),

(5) $M \vDash \neg F$,

(6) decisions $M \neq[]$,

then there is a state $\left(M^{\prime}, F^{\prime}\right)$ such that backjump' $(M, F)\left(M^{\prime}, F^{\prime}\right)$.

To ensure applicability of Lemma [5.9, the new version of the completeness theorem (Theorem 4.18) requires that the invariants hold in the current state. Since, by Lemma 5.5, backjump $(M, F)\left(M^{\prime}, F^{\prime}\right)$ implies backjump $(M, F)\left(M^{\prime}, F^{\prime}\right)$, the following completeness theorem holds for both transition systems presented in this section (using the rule backjump' or the rule backjump).

Theorem 5.10 (Completeness for $\left.\rightarrow_{b}\right)$. If $\left([], F_{0}\right) \rightarrow_{b}^{*}(M, F)$, and $(M, F)$ is a final state, then $(M, F)$ is either accepting or rejecting.

Proof. Let $(M, F)$ be a final state. By Lemma 4.6, all invariants hold in $(M, F)$. Also, it holds that either $M \vDash \neg F$ or $M \not \models \neg F$.

If $M \not \models \neg F$, since decide is not applicable, $(M, F)$ is an accepting state.

If $M \vDash \neg F$, assume that decisions $M \neq[]$. By Lemma [5.9, there is a state $\left(M^{\prime}, F^{\prime}\right)$ such that backjump $(M, F)\left(M^{\prime}, F^{\prime}\right)$. This contradicts the assumption that $(M, F)$ is a final state. Therefore, decisions $M=[]$, and since $M \vDash \neg F,(M, F)$ is a rejecting state.

Correctness of the system is a consequence of soundness, termination, and completeness, in analogy with Theorem 4.19 , 


\section{Learning And Forgetting}

In this section we briefly describe a system obtained from the system introduced in Section 5 by adding two new transition rules. These rules will have a significant role in more complex systems discussed in the following sections.

6.1. States and Rules. The relation $\rightarrow_{b}$ introduced in Section 5 is extended by the two following transition rules (introduced in the form of relations over states).

Definition 6.1 (Transition rules).

$$
\begin{aligned}
& \text { learn }\left(M_{1}, F_{1}\right)\left(M_{2}, F_{2}\right) \quad \text { iff } \quad \exists c . \quad F_{1} \vDash c \wedge \text { vars } c \subseteq \text { Vars } \wedge \\
& F_{2}=F_{1} @ c \wedge M_{2}=M_{1} \\
& \text { forget }\left(M_{1}, F_{1}\right)\left(M_{2}, F_{2}\right) \quad \text { iff } \quad \exists c . F_{1} \backslash c \vDash c \wedge \\
& F_{2}=F_{1} \backslash c \wedge M_{2}=M_{1}
\end{aligned}
$$

The extended transition system will be denoted by $\rightarrow_{l}$.

The learn rule is defined very generally. It is not specified how to construct the clause $c$ - typically, only clauses resulting from the conflict analysis process (Section 7) are learnt. This is the only rule so far that changes $F$, but the condition $F \vDash c$ ensures that it always remains logically equivalent to the initial formula $F_{0}$. The condition vars $c \subseteq$ Vars is relevant only for ensuring termination.

The forget rule changes the formula by removing a clause that is implied by all other clauses (i.e., is redundant). It is also not specified how this clause $c$ is chosen.

Example 6.2. Let $F_{0}$ be a formula from Example 4.5. A possible $\rightarrow_{l}$ trace is given by

\begin{tabular}{|c|c|c|}
\hline rule & $M$ & $F$ \\
\hline & & $F_{0}$ \\
\hline decide $(l=+1)$ & {$\left[+1^{d}\right]$} & $F_{0}$ \\
\hline unitPropagate $(c=[-1,+2], l=+2)$ & {$\left[+1^{d},+2^{i}\right]$} & $F_{0}$ \\
\hline unitPropagate $(c=[-2,+3], l=+3)$ & {$\left[+1^{d},+2^{i},+3^{i}\right]$} & $F_{0}$ \\
\hline decide $(l=+4)$ & {$\left[+1^{d},+2^{i},+3^{i},+4^{d}\right]$} & $F_{0}$ \\
\hline decide $(l=+5)$ & {$\left[+1^{d},+2^{i},+3^{i},+4^{d},+5^{d}\right]$} & $F_{0}$ \\
\hline unitPropagate $(c=[-5,+6], l=+6)$ & {$\left[+1^{d},+2^{i},+3^{i},+4^{d},+5^{d},+6^{i}\right]$} & $F_{0}$ \\
\hline unitPropagate $(c=[-2,-5,+7], l=+7)$ & {$\left[+1^{d},+2^{i},+3^{i},+4^{d},+5^{d},+6^{i},+7^{i}\right]$} & $F_{0}$ \\
\hline backjump $(c=[-2,-3,-5], l=-5)$ & {$\left[+1^{d},+2^{i},+3^{i},-5^{i}\right]$} & $F_{0}$ \\
\hline learn $(c=[-2,-3,-5])$ & {$\left[+1^{d},+2^{i},+3^{i},-5^{i}\right]$} & $F_{0} @[-2,-3,-5]$ \\
\hline unitPropagate $(c=[-1,-3,+5,+7], l=+7)$ & {$\left[+1^{d},+2^{i},+3^{i},-5^{i},+7^{i}\right]$} & $F_{0} @[-2,-3,-5]$ \\
\hline backjump $(c=[-1], l=-1)$ & {$\left[-1^{i}\right]$} & $F_{0} @[-2,-3,-5]$ \\
\hline decide $(l=+2)$ & {$\left[-1^{i},+2^{d}\right]$} & $F_{0} @[-2,-3,-5]$ \\
\hline unitPropagate $(c=[-2,+3], l=+3)$ & {$\left[-1^{i},+2^{d},+3^{i}\right]$} & $F_{0} @[-2,-3,-5]$ \\
\hline unitPropagate $(c=[-2,-3,-5], l=-5)$ & {$\left[-1^{i},+2^{d},+3^{i},-5^{i}\right]$} & $F_{0} @[-2,-3,-5]$ \\
\hline decide $(l=+4)$ & {$\left[-1^{i},+2^{d},+3^{i},-5^{i},+4^{d}\right]$} & $F_{0} @[-2,-3,-5]$ \\
\hline decide $(l=+6)$ & {$\left[-1^{i},+2^{d},+3^{i},-5^{i},+4^{d},+6^{d}\right]$} & $F_{0} @[-2,-3,-5]$ \\
\hline unitPropagate $(c=[-3,-6,-7], l=-7)$ & {$\left[-1^{i},+2^{d},+3^{i},-5^{i},+4^{d},+6^{d},-7^{i}\right]$} & $F_{0} @[-2,-3,-5]$ \\
\hline
\end{tabular}
(note that, unlike in the trace shown in Example 5.2, a clause $[-1,-2,-3]$ is learnt and used afterwards for unit propagation in another part of the search tree, eventually leading to a shorter trace): 
6.2. Properties. The new set of rules preserves all the invariants given in Section 4.2.1. Indeed, since learn and forget do not change the trail $M$, all invariants about the trail itself are trivially preserved by these rules. It can be proved that Invequiv, Inv varsF and InvimpliedLiterals also hold for the new rules.

Since the invariants are preserved in the new system, soundness is proved as in Theorem 4.9. Completeness trivially holds, since introducing new rules to a complete system cannot compromise its completeness. However, the extended system is not terminating since the learn and forget rules can by cyclically applied. Termination could be ensured with some additional restrictions. Specific learning, forgetting and backjumping strategies that ensure termination will be defined and discussed in Sections 7 and 8 .

\section{Conflict Analysis}

The backjumping rules, as defined in Section 5, are very general. If backjump clauses faithfully reflect the current conflict, they typically lead to significant pruning of the search space. In this section we will consider a transition system that employs conflict analysis in order to construct backjump clauses, which can be (in addition) immediately learned (by the rule learn).

7.1. States and Rules. The system with conflict analysis requires extending the definition of state introduced in Section 4 .

Definition 7.1 (State). A state of the system is a four-tuple $(M, F, C, c f l c t)$, where $M$ is a trail, $F$ is a formula, $C$ is a clause, and $c$ flct is a Boolean variable. A state $\left([], F_{0},[], \perp\right)$ is an initial state for the input formula $F_{0}$.

Two new transition rules conflict and explain are defined in the form of relations over states. In addition, the existing rules are updated to map four-tuple states to four-tuple states.

Definition 7.2 (Transition rules).

$$
\begin{aligned}
& \text { decide }\left(M_{1}, F_{1}, C_{1}, \text { cflct }_{1}\right)\left(M_{2}, F_{2}, C_{2}, \text { cflct }_{2}\right) \text { iff } \\
& \exists l . \quad \text { var } l \in \text { DecVars } \wedge l \notin M_{1} \wedge \bar{l} \notin M_{1} \wedge \\
& M_{2}=M_{1} @ l^{d} \wedge F_{2}=F_{1} \wedge C_{2}=C_{1} \wedge \text { cflct }_{2}=\text { cflct }_{1} \\
& \text { unitPropagate }\left(M_{1}, F_{1}, C_{1}, \text { cflct }_{1}\right)\left(M_{2}, F_{2}, C_{2}, \text { cflct }_{2}\right) \text { iff } \\
& \exists c l . \quad F_{1} \vDash c \wedge \operatorname{var} l \in \text { vars Vars } \wedge \text { isUnit } c l M_{1} \wedge \\
& M_{2}=M_{1} @ l^{i} \wedge F_{2}=F_{1} \wedge C_{2}=C_{1} \wedge \text { cflct }_{2}=\text { cflct }_{1} \\
& \text { conflict }\left(M_{1}, F_{1}, C_{1}, \text { cflct }_{1}\right)\left(M_{2}, F_{2}, C_{2}, \text { cflct }_{2}\right) \text { iff } \\
& \exists \text { c. } \quad \text { cflct }_{1}=\perp \wedge F_{1} \vDash c \wedge M_{1} \vDash \neg c \wedge \\
& M_{2}=M_{1} \wedge F_{2}=F_{1} \wedge C_{2}=c \wedge c_{c l c t}=\top \\
& \text { explain }\left(M_{1}, F_{1}, C_{1}, c f l c t_{1}\right)\left(M_{2}, F_{2}, C_{2}, \text { cflct }_{2}\right) \text { iff } \\
& \exists l c . \quad \text { cflct }_{1}=\top \wedge l \in C_{1} \wedge \text { isReason } c \bar{l} M_{1} \wedge F_{1} \vDash c \wedge \\
& M_{2}=M_{1} \wedge F_{2}=F_{1} \wedge C_{2}=\text { resolve } C_{1} c l \wedge \text { cflct }_{2}=\top \\
& \text { backjump }\left(M_{1}, F_{1}, C_{1}, c f l c t_{1}\right)\left(M_{2}, F_{2}, C_{2}, \text { cflct }_{2}\right) \text { iff }
\end{aligned}
$$




$$
\begin{aligned}
& \exists \text { l level. } \quad \text { cflct }_{1}=\top \wedge \text { isBackjumpLevel level } l C_{1} M_{1} \wedge \\
& M_{2}=\left(\text { prefixToLevel level } M_{1}\right) @ l^{i} \wedge F_{2}=F_{1} \wedge \\
& C_{2}=[] \wedge \text { cflct }_{2}=\perp \\
& \text { learn }\left(M_{1}, F_{1}, C_{1}, c f l c t_{1}\right)\left(M_{2}, F_{2}, C_{2}, c f l c t_{2}\right) \text { iff } \\
& \text { cflct }_{1}=\top \wedge C_{1} \notin F_{1} \\
& M_{2}=M_{1} \wedge F_{2}=F_{1} @ C_{1} \wedge C_{2}=C_{1} \wedge \text { cflct }_{2}=\text { cflct }_{1}
\end{aligned}
$$

The relation $\rightarrow_{c}$ is defined as in Definition 4.3 , but using the above list of rules. The definition of outcome states also has to be updated.

Definition 7.3 (Outcome states). A state is an accepting state if cflct $=\perp, M \not \models \neg F$ and there is no literal such that var $l \in$ DecVars, $l \notin M$ and $\bar{l} \notin M$.

A state is a rejecting state if cflct $=\top$ and $C=[]$.

Example 7.4. Let $F_{0}$ be a formula from Example 4.5. A possible $\rightarrow_{c}$ trace (shown up to the first application of backjump) is given (due to the lack of space, the $F$ component of the state is not shown).

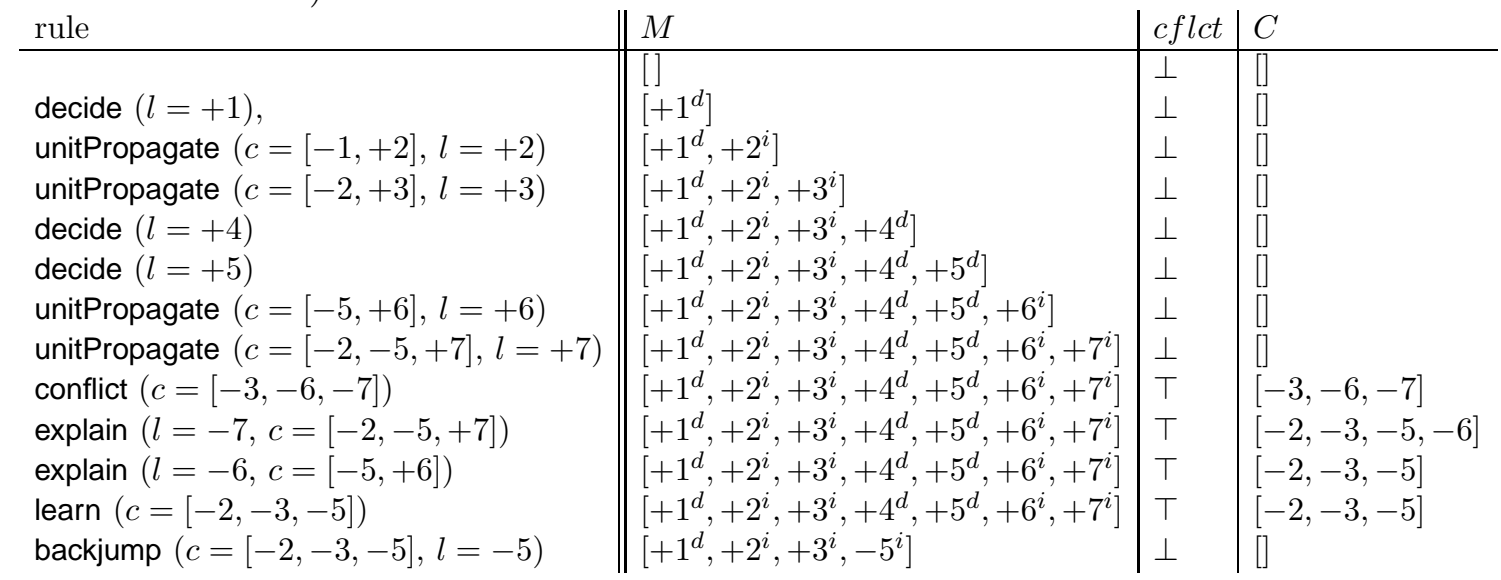

7.2. Unique Implication Points (UIP). SAT solvers employ different strategies for conflict analysis. The most widely used is a 1-UIP strategy, relying on a concept of unique implication points (UIP) (often expressed in terms of implication graphs [MSS99]). Informally, a clause $c$, false in the trail $M$, satisfies the UIP condition if there is exactly one literal in $c$ that is on the highest decision level of $M$. The UIP condition is very easy to check. The 1-UIP strategy requires that the rule explain is always applied to the last literal false in $M$ among literals from $c$, and that backjumping is applied as soon as $c$ satisfies the UIP condition.

Definition 7.5 (Unique implication point). A clause $c$ that is false in $M$ has a unique implication point, denoted by isUIP $l c M$, if the level of the last literal $l$ from $c$ that is false in $M$ is strictly greater than the level of the remaining literals from $c$ that are false in $M$ :

$$
\text { isUIP } l c M \quad \text { iff } \quad \begin{aligned}
& M \vDash \neg c \wedge \bar{l}=\text { lastAssertedLiteral } \bar{c} M \wedge \\
& \forall l^{\prime} . l^{\prime} \in c \backslash l \longrightarrow \text { level } \overline{l^{\prime}} M<\text { level } \bar{l} M
\end{aligned}
$$


The following lemma shows that, if there are decision literals in $M$, if a clause has a unique implication point, then there is a corresponding backjump level, and consequently, the backjump rule is applicable.

Lemma 7.6. If unique $M$ (i.e., Inv unique holds), then

$$
\text { isUIP } l \text { c } M \wedge \text { level } \bar{l} M>0 \text { iff } \exists \text { level. isBackjumpLevel level } l c M
$$

Therefore, the guard isBackjumpLevel level $l c M$ in the definition of the backjump rule can be replaced by the stronger conditions isUIP $l c M$ and level $\bar{l} M>0$. In that case, the backjump level level has to be explicitly calculated (as in the proof of the previous lemma).

The UIP condition is trivially satisfied when the clause $c$ consists only of opposites of decision literals from the trail (a similar construction of $c$ was already used in the proof of Lemma 5.9).

Lemma 7.7. If it holds that:

(1) unique $M$ (i.e., Inv unique holds),

(2) $\bar{c} \subseteq$ decisions $M$,

(3) $\bar{l}=$ lastAssertedLiteral $\bar{c} M$,

then isUIP $l c M$.

7.3. Properties. Properties of the new transition system will be again proved using invariants introduced in Section 4, but they have to be updated to reflect the new definition of states. In addition, three new invariants will be used.

7.3.1. Invariants. In addition to the invariants from Section 4, three new invariants are used.

$$
\begin{aligned}
& \text { Inv } \text { Ifalse: }_{\text {Inflat }} \longrightarrow M \vDash \neg C \\
& \text { Inv } \text { Centailed }: \quad \text { fflct } \longrightarrow F \vDash C \\
& \text { Inv }_{\text {reasonClauses }}: \forall l . l \in M \wedge l \notin \text { decisions } M \longrightarrow \exists c \text {. isReason } c l M \wedge F \vDash c
\end{aligned}
$$

The first two invariants ensure that during the conflict analysis process, the conflict analysis clause $C$ is a consequence of $F$ and that $C$ is false in $M$. The third invariant ensures existence of clauses that are reasons of literal propagation (these clauses enable application of the explain rule). By the rules unitPropagate and backjump literals are added to $M$ only as implied literals and in both cases propagation is performed using a clause that is a reason for propagation, so this clause can be associated to the implied literal, and afterwards used as its reason.

Lemma 4.6 again has to be updated to address new rules and its proof has to be modified to reflect the changes in the definition of the relation $\rightarrow_{c}$.

7.3.2. Soundness. Although the soundness proof for unsatisfiable formulae could be again based on Lemma 4.8, this time it will be proved in an alternative, simpler way (that does not rely on the invariant Inv $v_{\text {impliedLits }}$ ), that was not possible in previous sections.

Lemma 7.8. If there is a rejecting state $(M, F, C$, cflct) such that it holds

(1) $F \equiv F_{0}$, (i.e., Invequiv holds)

(2) cflct $\longrightarrow F \vDash C$ (i.e., Inv Centailed holds),

then $F_{0}$ is unsatisfiable (i.e., $\neg\left(\right.$ sat $\left.F_{0}\right)$ ). 
Theorem 7.9 (Soundness for $\left.\rightarrow_{c}\right)$. If $\left([], F_{0},[], \perp\right) \rightarrow_{c}^{*}(M, F, C$, cflct), then:

(1) If DecVars $\supseteq$ vars $F_{0}$ and $(M, F)$ is an accepting state, then the formula is $F_{0}$ satisfiable and $M$ is its model (i.e., sat $F_{0}$ and model $M F_{0}$ ).

(2) If $\left(M, F, C\right.$, cflct) is a rejecting state, then the formula $F_{0}$ is unsatisfiable (i.e., $\neg\left(\right.$ sat $\left.F_{0}\right)$ ).

Proof. By Lemma 4.6, all the invariants hold in the state $(M, F, C, c f l c t)$.

(1) All conditions of Lemma 4.7 are met (adapted to the new defintion of state), so sat $F_{0}$ and model $M F_{0}$.

(2) All conditions of Lemma 7.8 are met, so $\neg\left(\right.$ sat $\left.F_{0}\right)$.

7.3.3. Termination. Termination of the system with conflict analysis will be proved by using a suitable well-founded ordering that is compatible with the relation $\rightarrow_{c}$, i.e., an ordering $\succ$ such that $s \rightarrow_{c} s^{\prime}$ yields $s \succ s^{\prime}$, for any two states $s$ and $s^{\prime}$. This ordering will be constructed as a lexicographic combination of four simpler orderings, one for each state component.

The rules decide, unitPropagate, and backjump change $M$ and no other state components. If a state $s$ is in one of these relations with the state $s^{\prime}$ then $\left.M \succ_{\operatorname{tr}}\right|_{\text {Vars }} M^{\prime}$ (for the ordering $\left.\succ_{\text {tr }}\right|_{\text {Vars }}$, introduced in Section 4.2.3).

The ordering $\left.\succ_{\text {tr }}\right|_{\text {Vars }}$ cannot be used alone for proving termination of the system, since the rules conflict, explain, and learn do not change $M$ (and, hence, if a state $s$ is transformed into a state $s^{\prime}$ by one of these rules, then it does not hold that $\left.M \succ_{\operatorname{tr}}\right|_{\text {Vars }} M^{\prime}$ ). For each of these rules, a specific well-founded ordering will be constructed and it will be proved that these rules decrease state components with respect to those orderings.

The ordering $\succ_{\text {bool }}$ will be used for handling the state component cflct and the rule conflict (the rule explain changes the state component $\mathrm{cflct}$, but also the state component $C$, so it will be handled by another ordering). Given properties of the ordering $\succ_{\text {bool }}$ are proved trivially.

Definition $7.10\left(\succ_{\text {bool }}\right) \cdot b_{1} \succ_{\text {bool }} b_{2}$ iff $b_{1}=\perp \wedge b_{2}=\top$.

Lemma 7.11. If conflict $\left(M_{1}, F_{1}, C_{1}\right.$, cflct $\left.t_{1}\right)\left(M_{2}, F_{2}, C_{2}\right.$, cflct $\left.t_{2}\right)$, then cflct $_{1} \succ_{\text {bool }}$ cflct $_{2}$.

Lemma 7.12. The ordering $\succ_{\text {bool }}$ is well-founded.

An ordering over clauses (that are the third component of the states) should be constructed such that the rule explain decreases the state component $C$ with respect to that ordering. Informally, after each application of the rule explain, a literal $l$ of the clause $C$ that is (by Inv $v_{C f a l s e}$ ) false in $M$ is replaced by several other literals that are again false in $M$, but for them it holds that their opposite literals precede the literal $\bar{l}$ in $M$ (since reason clauses are used). Therefore, the ordering of literals in the trail $M$ defines an ordering of clauses false in $M$. The ordering over clauses will be a multiset extension of the relation $\prec_{M}$ induced by the ordering of literals in $M$ (Definition 3.1). Each explanation step removes a literal from $C$ and replaces it with several literals that precede it in $M$. To avoid multiple occurrences of a literal in $C$, duplicates are removed. Solvers usually perform this operation explicitly and maintain the condition that $C$ does not contain duplicates. However, our ordering does not require this restriction and termination is ensured even without it.

Definition $7.13\left(\succ_{\text {Cla }}^{M}\right)$. For a trail $M, C_{1} \succ_{\text {Cla }}^{M} C_{2}$ iff $\left\langle\right.$ remDups $\left.\overline{C_{2}}\right\rangle \prec_{M}^{\text {mult }}\left\langle\right.$ remDups $\left.\overline{C_{1}}\right\rangle$.

Lemma 7.14. For any trail $M$, the ordering $\succ_{\text {Cla }}^{M}$ is well-founded. 
The following lemma ensures that each explanation step decreases the conflict clause in the ordering $\succ_{\mathrm{Cla}}^{M}$, for the current trail $M$. This ensures that each application of the explain rule decreases the state with respect to this ordering.

Lemma 7.15. If $l \in C$ and isReason $c \bar{l} M$, then $C \succ_{\text {Cla }}^{M}$ resolve $C$ c $l$.

Lemma 7.16. If explain $\left(M, F, C_{1}\right.$, cflct) $\left(M, F, C_{2}\right.$, cflct $)$, then $C_{1} \succ_{\mathrm{Cla}}^{M} C_{2}$.

The rule learn changes the state component $F$ (i.e., it adds a clause to the formula) and it requires constructing an ordering over formulae.

Definition $7.17\left(\succ_{\text {Form }}^{C}\right.$ ). For any clause $C, F_{1} \succ_{\text {Form }}^{C} F_{2}$ iff $C \notin F_{1} \wedge C \in F_{2}$.

Lemma 7.18. For any clause $C$, the ordering $\succ_{\text {Form }}^{C}$ is well-founded.

By the definition of the learn rule, it holds that $C \notin F_{1}$ and $C \in F_{2}$, so the following lemma trivially holds.

Lemma 7.19. If learn $\left(M, F_{1}, C\right.$, cflct) $\left(M, F_{2}, C\right.$, cflct $)$, then $F_{1} \succ_{\text {Form }}^{C} F_{2}$.

Theorem 7.20 (Termination for $\rightarrow_{c}$ ). If the set DecVars is finite, for any formula $F_{0}$, the relation $\rightarrow_{c}$ is well-founded on the set of states $s$ such that $s_{0} \rightarrow_{c}^{*} s$, where $s_{0}$ is the initial state for $F_{0}$.

Proof. Let $\succ$ be a (parametrized) lexicographic product (Definition ?), i.e., let

$$
\left.\succ \equiv \succ_{\text {tr }}\right|_{\text {Vars }}\langle * \operatorname{lex} *\rangle \succ_{\text {bool }}\left\langle * \operatorname{lex}^{\mathrm{p}} *\right\rangle\left(\lambda s . \succ_{\text {Cla }}^{M_{s}}\right)\left\langle * \operatorname{lex}^{\mathrm{p}} *\right\rangle\left(\lambda s . \succ_{\text {Form }}^{C_{s}}\right),
$$

where $M_{s}$ is the trail in the state $s$, and $C_{s}$ is the conflict clause in the state $s$. By Proposition 3.4 and Lemmas 4.16, 7.12, 7.14, and 7.18, the relation $\succ$ is well-founded. If the invariants hold in the state $\left(M_{1}, F_{1}, C_{1}\right.$, cflct $\left.t_{1}\right)$ and if $\left(M_{1}, F_{1}, C_{1}\right.$, cflct $\left._{1}\right) \rightarrow_{c}\left(M_{2}, F_{2}, C_{2}\right.$, cflct $\left._{2}\right)$, then $\left(M_{1}\right.$, cflct $\left._{1}, C_{1}, F_{1}\right) \succ\left(M_{2}\right.$, cflct $\left._{2}, C_{2}, F_{2}\right)$. Indeed, by Lemma 4.14, the rules decide, unitPropagate and backjump decrease $M$ in the ordering, the rule conflict does not change $M$ but (by Lemma 7.11) decreases cflct, the rule explain does not change $M$ nor $c$ flct, but (by Lemma 7.16) decreases $C$, and the rule learn does not change $M$, cflct, nor $C$, but (by Lemma 7.19) decreases $F$.

Then the theorem holds by Proposition 3.4 (where $\mathrm{f}$ is a permutation mapping $(M, F$, $C$, cflct) to $(M$, cflct $, C, F))$.

7.3.4. Completeness and Correctness. Completeness requires that all final states are outcome states, and the following two lemmas are used to prove this property.

Lemma 7.21. If for the state $(M, F, C$, cflct $)$ it holds that:

(1) cflct $=\top$,

(2) unique $M$ (i.e., Inv $v_{\text {unique }}$ holds),

(3) cflct $\longrightarrow M \vDash \neg C$ (i.e., Inv Cfalse holds),

(4) the rules explain and backjump are not applicable,

then the state $(M, F, C$, cflct $)$ is a rejecting state and $C=[]$.

Lemma 7.22. If in the state $(M, F, C$, cflct $)$ it holds that cflct $=\perp$ and the rule conflict $i s$ not applicable, then the state $(M, F, C$, cflct $)$ is an accepting state and $M \not \models \neg F$.

Theorem 7.23 (Completeness for $\rightarrow_{c}$ ). For any formula $F_{0}$, if $\left([], F_{0},[], \perp\right) \rightarrow_{c}^{*}(M, F, C, c f l c t)$, and if the state $(M, F, C$, cflct $)$ is final, then it is either accepting or rejecting. 
Proof. Since the state $(M, F, C$, cflct $)$ is reachable from the initial state, by Lemma 4.6, all the invariants hold in this state, including unique $M$ (i.e., Inv unique), and cflct $\longrightarrow M \vDash \neg C$ (i.e., Inv Cfalse $)$. In the state $(M, F, C$, cflct), it holds that either $c$ flct $=\top$ or $c$ flct $=\perp$. If cflct $=\perp$, since the rule decide is not applicable (as the state is final), by Lemma 7.22, the state $(M, F, C, c f l c t)$ is a rejecting state. If $c f l c t=\top$, since the rule conflict is not applicable (as the state is final) by Lemma 7.21, the state is an accepting state.

Correctness of the system is proved in analogy with Theorem 4.19,

\section{Restarting And Forgetting}

In this section we extend the previous system with restarting and forgetting. The most challenging task with restarting is to ensure termination.

Many solvers use restarting and forgetting schemes that apply restarting with increasing periodicity and there are theoretical results ensuring total correctness of these [KG07, NOT06]. However, modern solvers also use aggressive restarting schemes (e.g., Luby restarts) that apply the restart rule very frequently, but there are no corresponding theoretical results that ensure termination of these schemes. In this section we will formulate a system that allows application of the restart rule after each conflict and show that this (weakly constrained, hence potentially extremely frequent) scheme also ensures termination.

8.1. States and Rules. Unlike previous systems that tend to be as abstract as possible, this system aims to precisely describe the behaviour of modern SAT solvers. For example, only learnt clauses can be forgotten. So, to aid the forget rule, the formula is split to the initial part $F_{0}$ and the learnt clauses $F l$. Since the input formula $F_{0}$ is fixed it is not a part of state anymore, but rather an input parameter. The new component of the state - the lnt flag - has a role in ensuring termination by preventing applying restart and forget twice without learning a clause in between. In addition, some changes in the rules ensure termination of some variants of the system. Unit propagation is performed eagerly, i.e., decide is not applied when there is a unit clause present. Also, backjumping is always performed to the minimal backjump level (Definition [5.2). These stronger conditions are very often obeyed in real SAT solver implementations, and so this system still makes their faithful model.

Definition 8.1 (State). A state of the system is a five-tuple $(M, F l, C$, cflct, lnt $)$, where $M$ is a trail, $F l$ is a formula, $C$ is a clause, and $c f l c t$ and $l n t$ are Boolean variables. A state ([], $\left.F_{0},[], \perp, \perp\right)$ is a initial state for the input formula $F_{0}$.

Definition 8.2 (Transition rules).

$$
\begin{aligned}
& \text { decide }\left(M_{1}, F l_{1}, C_{1}, c f l c t_{1}, \operatorname{lnt}_{1}\right)\left(M_{2}, F l_{2}, C_{2}, c f l c t_{2}, \operatorname{lnt}_{2}\right) \text { iff } \\
& \exists l . \quad \operatorname{var} l \in \text { DecVars } \wedge l \notin M_{1} \wedge \bar{l} \notin M_{1} \wedge \\
& \neg\left(\exists c l . c \in F_{0} @ F l_{1} \wedge \text { isUnitClause } c \mid M_{1}\right) \wedge \\
& M_{2}=M_{1} @ l^{d} \wedge F l_{2}=F l_{1} \wedge C_{2}=C_{1} \wedge \text { cflct }_{2}=\text { cflct }_{1} \wedge \text { lnt }_{2}=\operatorname{lnt}_{1} \\
& \text { unitPropagate }\left(M_{1}, F l_{1}, C_{1}, c f l c t_{1}, \operatorname{lnt}_{1}\right)\left(M_{2}, F l_{2}, C_{2}, c f l c t_{2}, \text { lnt }_{2}\right) \text { iff } \\
& \exists c l . \quad c \in F_{0} @ F l_{1} \wedge \text { isUnit } c l M_{1} \wedge \\
& M_{2}=M_{1} @ l^{i} \wedge F l_{2}=F l_{1} \wedge C_{2}=C_{1} \wedge \text { cflct }_{2}=\text { cflct }_{1} \wedge \text { lnt }_{2}=\operatorname{lnt}_{1}
\end{aligned}
$$




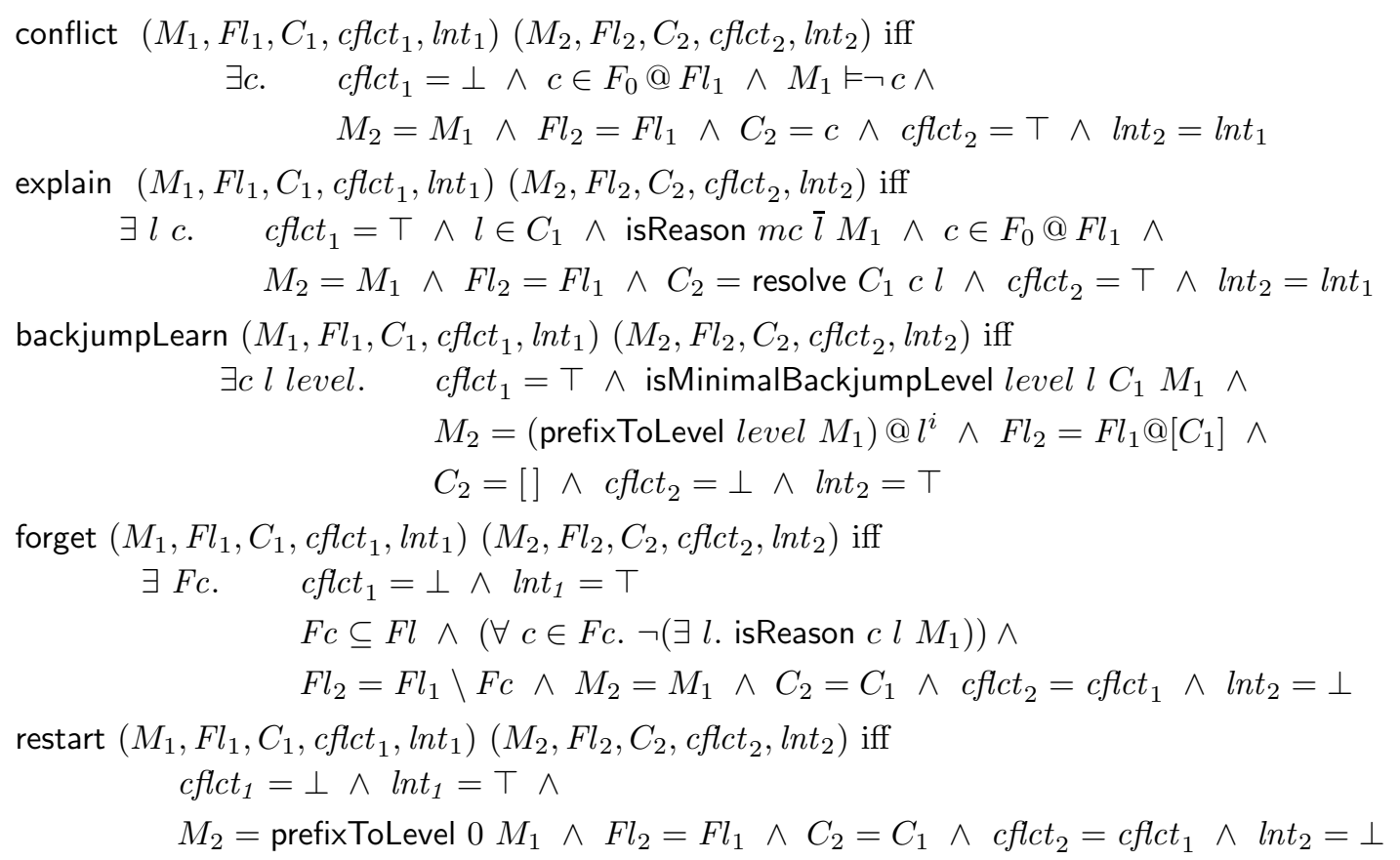

These rules will be used to formulate three different transition systems. The system $\rightarrow_{r}$ consists of all rules except restart, the system $\rightarrow_{f}$ consists of all rules except forget, and the system $\rightarrow$ consists of all rules.

8.2. Properties. The structure of the invariants and the proofs of the properties of the system are basically similar to those given in Section 7 , while the termination proof requires a number of new insights.

8.2.1. Invariants. All invariants formulated so far hold, but the formula $F$, not present in the new state, has to be replaced by $F_{0} @ F l$.

8.2.2. Termination. Termination of the system without restarts is proved first.

Theorem 8.3 (Termination for $\rightarrow_{r}$ ). If the set DecVars is finite, for any formula $F_{0}$, the relation $\rightarrow_{r}$ is well-founded on the set of states $s$ such that $s_{0} \rightarrow_{r}^{*} s$, where $s_{0}$ is the initial state for $F_{0}$.

Proof. Let $\succ$ be a (parametrized) lexicographic product (Definition ?), i.e., let

$$
\left.\succ \equiv \succ_{\text {tr }}\right|_{\text {Vars }}\langle * \operatorname{lex} *\rangle \succ_{\text {bool }}\left\langle * \operatorname{lex}^{\mathrm{p}} *\right\rangle\left(\lambda s . \succ_{\text {Cla }}^{M_{s}}\right)\langle * \operatorname{lex} *\rangle \succ_{\text {bool }},
$$

where $M_{s}$ is the trail in the state $s$. By Proposition 3.4 and Lemmas 4.16, 7.12, and 7.14, the relation $\succ$ is well-founded. If the state $\left(M_{1}, F l_{1}, C_{1}, c f l c t_{1}, l n t_{1}\right)$ satisfies the invariants and if $\left(M_{1}, F l_{1}, C_{1}, c f l c t_{1}, \operatorname{lnt}_{1}\right) \rightarrow_{r}\left(M_{2}, F l_{2}, C_{2}, c f l c t_{2}, \operatorname{lnt}_{2}\right)$, then $\left(M_{1}\right.$, cflct $\left._{1}, C_{1}, \neg \operatorname{lnt}_{1}\right) \succ$ $\left(M_{2}\right.$, cflct $\left._{2}, C_{2}, \neg l n t_{2}\right)$. Indeed, by Lemma 4.14 the rules unitPropagate, decide and backjumpLearn decrease $M$, the rule conflict does not change $M$ but (by Lemma 7.11) decreases cflct, the rule explain does not change $M$ nor cflct, but (by Lemma 7.16) decreases $C$, and the rule forget does not change $M$, cflct, nor $C$, but decreases $\neg \operatorname{lnt}$.

From the above, the theorem holds by Proposition 3.4 (for a suitable f). 
The termination proof of the system without forgets is more involved. We define a (not necessarily well-founded) ordering of the formulae by inclusion and its restriction with respect to the set of variables occurring in the formula.

Definition 8.4 ( $\left.\succ_{\text {Form } \subset}\right)$. $F_{1} \succ_{\text {Form } ~} F_{2}$ iff $F_{1} \subset F_{2}$.

Definition $8.5\left(\succ_{\text {Form }}||_{V b l}\right) .\left.F_{1} \succ_{\text {Form } \subset}\right|_{V b l} F_{2}$ iff vars $F_{1} \subseteq V l b \wedge$ vars $F_{1} \subseteq V b l \wedge$ $\overline{F_{1}} \succ_{\text {Form }} \overline{F_{2}}$, where $\bar{F}$ denotes the formula obtained by removing duplicate literals from clauses and removing duplicate clauses.

Lemma 8.6. If the set Vbl is finite, then the relation $\left.\succ_{\text {Formc }}\right|_{V b l}$ is well-founded.

The following lemma states that if unit propagation is done eagerly and if backjumping is always performed to the minimal backjump level, then the clauses that are learnt are always fresh, i.e., they do not belong to the current formula.

Lemma 8.7. If $s_{0}$ is an initial state, $s_{0} \rightarrow_{f}^{*} s_{A}$ and backjumpLearn $s_{A} s_{B}$, where $s_{A}=$ $\left(M_{A}, F l_{A}, C_{A}, \top, \ln t_{A}\right)$, then $C_{A} \notin F_{0} @ F l_{A}$.

Therefore, backjumpLearn increases formula in the inclusion ordering.

Lemma 8.8. If $s_{0} \rightarrow_{f} s_{A}$ and backjumpLearn $s_{A} s_{B}$ for initial state $s_{0}$ and states $s_{A}$ and $s_{B}$, then $\left.F_{0} @ F l_{A} \succ_{\text {Form }}\right|_{V a r s} F_{0} @ F l_{B}$, where $F_{A}$ and $F_{B}$ are formulae in states $s_{A}$ and $s_{B}$.

Theorem 8.9 (Termination for $\rightarrow_{f}$ ). If the set DecVars is finite, for any formula $F_{0}$, the relation $\rightarrow_{f}$ is well-founded on the set of states s such that $s_{0} \rightarrow_{f}^{*} s$, where $s_{0}$ is the initial state for $F_{0}$.

Proof. Let $\succ$ be a (parametrized) lexicographic product (Definition ?), i.e., let

$\left.\succ \equiv \succ_{\text {Formc }}\right|_{\text {Vars }}\langle * \operatorname{lex} *\rangle \succ_{\text {bool }}\langle * \operatorname{lex} *\rangle \succ_{\text {tr }} \mid$ Vars $\langle * \operatorname{lex} *\rangle \succ_{\text {bool }}\left\langle * \operatorname{lex}^{\mathrm{p}} *\right\rangle\left(\lambda s . \succ_{\text {Cla }}^{M_{s}}\right)$,

where $M_{s}$ is the trail in the state $s$. By Proposition 3.4 and Lemmas 4.16, 7.12, 7.14, and 8.6, the relation $\succ$ is well-founded. If the state $\left(M_{1}, F l_{1}, C_{1}, c f l c t_{1}, l n t_{1}\right)$ satisfies the invariants and if $\left(M_{1}, F l_{1}, C_{1}\right.$, cflct $\left._{1}, \operatorname{lnt}_{1}\right) \rightarrow_{f}\left(M_{1}, F l_{1}, C_{1}\right.$, cflct $\left._{1}, \operatorname{lnt}_{1}\right)$, then $\left(F_{1}, \neg \operatorname{lnt}_{1}, M_{1}\right.$, cflct $\left._{1}, C_{1}\right) \succ$ $\left(F_{2}, \neg l n t_{2}, M_{2}\right.$, cflct $\left._{2}, C_{2}\right)$. Indeed, by Lemma 8.8 the rule backjumpLearn decreases $F$, the rule restart does not change $F$ but decreases $\neg \ln t$, the rules unitPropagate and decide do not change $F$ and $l n t$ but (by Lemma 4.14) decrease $M$, the rule conflict does not change $F$, lnt, nor $M$ but (by Lemma 7.11) decreases cflct, and the rule explain does not change $F$, lnt, $M$ nor cflct, but (by Lemma 7.16) decreases $C$.

From the above, the theorem holds by Proposition 3.4 (for a suitable f).

If both forget and restart are allowed, then the system is not terminating.

Theorem 8.10. The relation $\rightarrow$ is not well-founded on the set of states reachable from the initial state.

Proof. Consider the formula $[[-1,-2,3],[-1,-2,4],[-1,-3,-4],[-5,-6,7],[-5,-6,8]$, $[-5,-7,-8]]$. The following derivation chain (for simplicity, not all components of the states are shown) proves that the relation $\rightarrow$ is cyclic. 


\begin{tabular}{|c|c|c|c|}
\hline rule & $M$ & $\mathrm{Fl}$ & $\ln t$ \\
\hline & & & $\perp$ \\
\hline decide, decide & {$\left[1^{d}, 2^{d}\right]$} & & $\perp$ \\
\hline unitPropagate, unitPropagate & {$\left[1^{d}, 2^{d}, 3^{i}, 4^{i}\right]$} & & $\perp$ \\
\hline conflict, explain, explain, backjumpLearn & {$\left[1^{d},-2^{i}\right]$} & {$[[-1,-2]]$} & $\top$ \\
\hline restart & & {$[[-1,-2]]$} & $\perp$ \\
\hline decide, decide & {$\left[5^{d}, 6^{d}\right]$} & {$[[-1,-2]]$} & $\perp$ \\
\hline unitPropagate, unitPropagate & {$\left[5^{d}, 6^{d}, 7^{d}, 8^{d}\right]$} & {$[[-1,-2]]$} & $\perp$ \\
\hline conflict, explain, explain, backjumpLearn & {$\left[5^{d},-6^{d}\right]$} & {$[[-1,-2],[-5,-6]]$} & $\top$ \\
\hline forget & {$\left[5^{d},-6^{i}\right]$} & & $\perp$ \\
\hline decide, decide & {$\left[5^{d},-6^{i}, 1^{d}, 2^{d}\right]$} & & $\perp$ \\
\hline unitPropagate, unitPropagate & {$\left[5^{d},-6^{i}, 1^{d}, 2^{d}, 3^{i}, 4^{i}\right]$} & & $\perp$ \\
\hline conflict, explain, explain, backjumpLearn & {$\left[5^{d},-6^{i}, 1^{d},-2^{i}\right]$} & {$[[-1,-2]]$} & T \\
\hline restart & & {$[[-1,-2]]$} & $\perp$ \\
\hline
\end{tabular}

Therefore, it holds that

$$
([],[],[], \perp, \perp) \rightarrow^{*}([],[[-1,-2]],[], \perp, \perp) \rightarrow^{+}([],[[-1,-2]],[], \perp, \perp) .
$$

However, if there are additional restrictions on the rule application policy, the system may be terminating. Since the number of different states for the input formula $F_{0}$ is finite (when duplicate clauses and literals are removed), there is a number $n_{f}$ (dependent on $\left.F_{0}\right)$ such that there is no chain of rule applications without forget longer than $n_{f}\left(\rightarrow_{f}\right.$ is well-founded and therefore acyclic, so, on a finite set, there must exist $n_{f}$ such that $\rightarrow_{f}^{n_{f}}$ is empty). Similarly, there is a number $n_{r}$ (dependent on $F_{0}$ ) such that there is no chain of rule applications without restart longer than $n_{r}$. So, termination is ensured for any policy that guarantees that there is a point where the application of forget will be forbidden for at least $n_{f}$ steps or that there is a point where the application of restart will be forbidden for at least $n_{r}$ steps.

8.2.3. Soundness, Completeness and Correctness. Soundness and completeness proofs from previous sections hold with minor modifications necessary to adapt them to the new definition of state and rules. The most demanding part is to update Lemma 4.6 and to prove that the new rules maintain the invariants.

\section{Related Work And Discussions}

The original DPLL procedure DLL62 has been described in many logic textbooks, along with informal proofs of its correctness (e.g., [DSW94]). First steps towards verification of modern DPLL-based SAT solvers have been made only recently. Zhang and Malik have informally proved correctness of a modern SAT solver ZM03. Their proof is very informal, the specification of the solver is given in pseudo-code and it describes only one strategy for applying rules. The authors of two abstract transition systems for SAT also give correctness proofs [NOT06, KG07]. These specifications and the proofs are much more formal than those given in [ZM03, but they are also not machine-verifiable and are much less rigorous than the proofs presented in this paper. 
In recent years, several machine-verifiable correctness proofs for SAT solvers were constructed. Lescuyer and Conchon formalized, within Coq, a SAT solver based on the classical DPLL procedure and its correctness proof [LS08]. They used a deep embedding, so this approach enables execution of the SAT solver in Coq and, further, a reflexive tactic. Marić and Janičić formalized a correctness proof for the classical DPLL procedure by shallow embedding into Isabelle/HOL [MJ10]. Shankar and Vaucher formally and mechanically verified a high-level description of a modern DPLL-based SAT solver within the system PVS SV09]. However, unlike this paper which formalizes abstract descriptions for SAT, they formalize a very specific SAT solver implementation within PVS. Marić proved partial correctness (termination was not discussed) of an imperative pseudo-code of a modern SAT solver using Hoare logic approach [Mar09] and total correctness of a SAT solver implemented in Isabelle/HOL using shallow embedding [Mar10. Both these formalizations use features of the transition systems described in this paper and provide links between the transition systems and executable implementations of modern SAT solvers. In the former approach, the verified specification can be rewritten to an executable code in an imperative programming language 11 while in the latter approach, an executable code in a functional language can be exported from the specification by automatic means [HN10].

The transition system discussed in Section 4 corresponds to a non-recursive version of the classical DPLL procedure. The transition systems and correctness proofs presented in the later sections are closely related to the systems of Nieuwenhuis et al. [NOT06] and Krstić and Goel [KG07]. However, there are some significant differences, both in the level of precision in the proofs and in the definitions of the rules.

Informal (non machine-verifiable) proofs allow authors some degree of imprecision. For example, in [NOT06] and [KG07] clauses are defined as "disjunctions of literals" and formulae as "conjunctions of clauses", and this leaves unclear some issues such as whether duplicates are allowed. The ordering of clauses and literals is considered to be irrelevant in [KG07] it is said that "clauses containing the same literals in different order are considered equal", and in [NOT06] it is not explicitly said, but only implied (e.g., clauses in the unitPropagate rule are written as $C \vee l$, where $M \vDash \neg C$ and $l$ is undefined in $M$, and from this it is clear that the order of literals must be irrelevant, or otherwise only last literals in clauses could be propagated). Therefore, clauses and formulae are basically defined as sets or multisets of literals. In our formal definition, clauses and formulae are defined as lists. Although a choice whether to use lists, multisets, or sets in these basic definitions might not seem so important, fully formal proofs show that this choice makes a very big difference. Namely, using sets saves much effort in the proof. For example, if formulae may contain repeated clauses, easy termination arguments like "there are finitely many different clauses that can be learnt" cannot be applied. On the other hand, using sets makes the systems quite different from real SAT solver implementations - eliminating duplicates from clauses during solving is possible and cheap, but explicitly maintaining absence of duplicate clauses from formulae may be intolerably expensive. It can be proved that maintaining absence of duplicate clauses can be, under some conditions on the rules, implicitly guaranteed only by eliminating duplicate clauses from formulae during initialization. Solvers typically assume this complex fact, but it was not proved before for formulae represented by lists, while for systems using sets this issue is irrelevant.

\footnotetext{
${ }^{11}$ As done in the implementation of our SAT solver ArgoSAT.
} 
The system given in NOT06 is very close to the system given in Section 5 and later extended in Section 6. The requirement that the set of decision literals exactly coincides with the set of literals from the input formula is too strong and is not always present in real SAT solvers, so it is relaxed in our system and the set DecVars is introduced (a similar technique is used in [KG07]). Also, the definition of the backjump rule from [NOT06] requires that there is a false clause in the formula being solved when the rule is applied, but our formal analysis of the proofs shows that this assumption is not required, so it is omitted from Definition 5.1. As already mentioned, the condition that the unit clauses belong to the formula is also relaxed, and propagating can be performed over arbitrary consequences of the formula. The invariants used in the proofs and the soundness proof are basically the same in [NOT06] and in this paper, but the amount of details had to be significantly increased to reach a machine-verifiable proof. Our completeness proof is somewhat simpler. The ordering used in termination proof for the system with backjumping in [NOT06] expresses a similar idea to ours, but is much more complex. A conflict analysis process is not described within the system from [NOT06.

The system given in [KG07] is close to the system given in Section [7, with some minor differences. Namely, in our system, instead of a set of decision literals, the set of decision variables is considered. Also, unit, conflict and reason clauses need not be present in the formula. The conflict set used in [KG07] along with its distinguished value no_cflct is here replaced by the conflict flag and a conflict clause (the conflict set is the set of opposites of literals occurring in our conflict clauses). The underlying reasoning used in two total correctness proofs is the same, although in [KG07] the invariants are not explicitly formulated and the proof is monolithic (lemmas are not present) and rather informal.

Formalization of termination proofs from both [NOT06] and [KG07] required the greatest effort in the formalization. Although arguments like "between any two applications of the rule ... there must be an occurrence of the rule ...", heavily used in informal termination proofs, could be formalized, we felt that constructing explicit termination orderings is much cleaner.

In KG07 termination of systems with restarts is not thoroughly discussed and in [NOT06] it is proved very informally, under a strong condition that the periodicity of restarts is strictly increasing. This is often not the case in many modern SAT solver implementations. In this paper, we have (formally) proved that restarting can be performed very frequently (after each conflict) without compromising total correctness. However, some additional requirements (unit propagation must be exhaustive, backjumping must be performed to minimal backjumping levels, and backjump lemmas must always be learnt) are used in the proof, but these are always present in modern SAT solvers. Although the issue has been addressed in the literature, we are not aware of a previous proof of termination of frequent restarting.

\section{Conclusions}

We presented a formalization of modern SAT solvers and their properties in the form of abstract state transition systems. Several different SAT solvers are formalized - from the classical DPLL procedure to its modern successors. The systems are defined in a very abstract way so they cover a wide range of SAT solving procedures. The formalization is made within the Isabelle/HOL system and the total correctness properties (soundness, termination, completeness) are shown for each presented system. 
Central theorems claim (roughly) that a transition system, i.e., a SAT solver, terminates and returns an answer yes if and only if the input formula is satisfiable. This whole construction boils down to the simple definition of satisfiable formula, which can be confirmed by manual inspection.

Our formalization builds up on the previous work on state transition systems for SAT and also on correctness arguments for other SAT systems. However, our formalization is the first that gives machine-verifiable total correctness proofs for systems that are close to modern SAT solvers. Also, compared to other abstract descriptions, our systems are more general (so can cover a wider range of possible solvers) and require weaker assumptions that ensure the correctness properties. Thanks to the framework of formalized mathematics, we explicitly separated notions of soundness and completeness, and defined all notions and properties relevant for SAT solving, often neglected to some extent in informal presentations.

Our experience in the SAT verification project shows that having imperative software modelled abstractly, in the form of abstract state transition systems, makes the verification cleaner and more flexible. It can be used as a key building block in proving correctness of SAT solvers by using other verification approaches which significantly simplifies the overall verification effort.

\section{ACKNOWLEDGEMENT}

This work was partially supported by the Serbian Ministry of Science grant 174021 and by the SNF grant SCOPES IZ73Z0_127979/1. We are grateful to Natarajan Shankar for sharing with us his unpublished manuscript [SV09]. We are also grateful to anonymous reviewers for very careful reading and for detailed and useful comments on an earlier version of this paper.

\section{REFERENCES}

[BMS00] L. Baptista and J. P. Marques-Silva. Using randomization and learning to solve hard real-world instances of satisfiability. In 6th CP, Singapore, 2000.

[BS97] R. J. Jr. Bayardo and R. C. Schrag. Using CSP look-back techniques to solve real-world SAT instances. In 14th AAAI, Providence, USA, 1997.

[Bie08] A. Biere. PicoSAT Essentials. In JSAT, vol. 4, pp. 75-97, 2008.

[BHMW09] A. Biere, M. Heule, H. van Maaren, and T. Walsh editors. Handbook of satisfiability, IOS Press, 2009 .

[BHZ06] L. Bordeaux, Y. Hamadi, and L. Zhang. Propositional satisfiability and constraint programming: A comparative survey. In ACM Surveys, 2006.

$\left[\mathrm{BKH}^{+} 08\right]$ L. Bulwahn, A. Krauss, F. Haftmann, L. Erkök and J. Matthews. Imperative Functional Programming with Isabelle/HOL. In TPHOLs 2008, Montreal, LNCS 5170, 2008.

[Coo71] S. A. Cook. The complexity of theorem-proving procedures. In 3rd STOC, New York, USA, 1971.

[DFMS10] A. Darbari, B. Fischer, J. Marques-Silva. Industrial-Strength Certified SAT Solving through Verified SAT Proof Checking. In ICTAC, 2010.

[DLL62] M. Davis, G. Logemann, and D. Loveland. A machine program for theorem-proving. Commun. $A C M, 5(7), 1962$.

[DP60] M. Davis and H. Putnam. A computing procedure for quantification theory. J. ACM, 7(3), 1960.

[DSW94] M. Davis, R. Sigal, E. J Weyuker. Computability, Complexity, and Languages: Fundamentals of Theoretical Computer Science. Morgan Kaufmann Publishers, 1994.

[ES04] N. Eén and N. Sorensson. An extensible SAT-solver. in SAT 04, 2004.

[Gel07] A. Van Gelder. Verifying Propositional Unsatisfiability: Pitfalls to Avoid. In SAT 'O7, LNCS 4501, Lisbon, 2007. 
[GKSS07] C P. Gomes, H. Kautz, A. Sabharwal, and B. Selman. Satisfiability solvers. In Handbook of Knowledge Representation. Elsevier, 2007.

[GN02] E. Goldberg and Y. Novikov. Berkmin: A fast and robust SAT solver. In Design Automation and Test in Europe (DATE), 2002.

[GN03] E. Goldberg and Y. Novikov. Verification of Proofs of Unsatisfiability for CNF Formulas. In Design Automation and Test in Europe (DATE), 2003.

[GSK98] C. Gomes, B. Selman, and H. Kautz. Boosting combinatorial search through randomization. In 15th AAAI, Madison, WI, USA, 1998.

[HN10] F. Haftmann, T. Nipkow. Code Generation via Higher-Order Rewrite Systems. In FLOPS 2010, LNCS 6009, Springer, 2010.

[Hoa69] C. A. R. Hoare. An axiomatic basis for computer programming. Communication of the ACM, 12(10), 1969.

[Kle10] G. Klein et al. seL4: formal verification of an operating-system kernel. Communication of the $A C M, 53(6), 2010$.

[KG07] S. Krstić and A. Goel. Architecting solvers for SAT modulo theories: Nelson-Oppen with DPLL. In FroCoS, 2007.

[LS08] S. Lescuyer and S. Conchon A Reflexive Formalization of a SAT Solver in Coq. In TPHOLs'08: Emerging Trends, Montreal, 2008.

[Mar08] F. Marić, SAT Solver Verification. The Archive of Formal Proofs, http://afp.sf.net/entries/SATSolverVerification.shtml.

[Mar09] F. Marić. Formalization and Implementation of SAT solvers. J. Autom. Reason., 43(1), 2009.

[Mar10] F. Marić. Formal Verification of a Modern SAT Solver by shallow embedding into Isabelle/HOL. Theoretical Computer Science, 411(50), 2010.

[MJ09] F. Marić and P. Janičić. SAT Verification Project. In TPHOLs'09: Emerging Trends, Munich, 2009.

[MJ10] F. Marić and P. Janičić. Formal Correctness Proof for DPLL Procedure. Informatica, 21(1), 2010 .

[MSS99] J P. Marques-Silva and K A. Sakallah. Grasp: A new search algorithm for satisfiability. In International Conference on Computer-Aided Design, 1996.

$\left[\mathrm{MMZ}^{+} 01\right]$ M. W. Moskewicz, C. F. Madigan, Y. Zhao, L. Zhang, and S. Malik. Chaff: Engineering an Efficient SAT Solver. In 38th DAC, 2001.

[NOT06] R. Nieuwenhuis, A. Oliveras, and C. Tinelli. Solving SAT and SAT Modulo Theories: from an Abstract Davis-Putnam-Logemann-Loveland Procedure to DPLL(T). Journal of the ACM, 53(6), 2006.

[NPW02] T. Nipkow, L. C. Paulson, and M. Wenzel. Isabelle/HOL - A Proof Assistant for Higher-Order Logic, LNCS 2283, Springer, 2002.

[Sch06] N. Schirmer. Verification of Sequential Imperative Programs in Isabelle/HOL. PhD thesis, Technische Universität München (2006).

[SV09] N. Shankar and M. Vaucher. The mechanical verification of a DPLL-based satisfiability solver. Unpublished manuscript, 2008.

[WA09] T. Weber and H. Amjad. Efficiently checking propositional refutations in HOL theorem provers. J. Applied Logic 7(1), 2009.

[Zha97] H. Zhang. SATO: An efficient propositional prover. In CADE-14, London, UK, 1997.

[ZM02] L. Zhang and S. Malik. The quest for efficient boolean satisfiability solvers. In 14 th $C A V$, London, UK, 2002.

[ZM03] L. Zhang and S. Malik. Validating SAT solvers using independent resolution-based checker. In DATE'03, Washington DC, USA, 2003.

[ZMMM01] L. Zhang, C. F. Madigan, M. H. Moskewicz, and S. Malik. Efficient conflict driven learning in a boolean satisfiability solver. In ICCAD '01, Piscataway, USA, 2001.

This work is licensed under the Creative Commons Attribution-NoDerivs License. To view a copy of this license, visit http://creativecommons.org/licenses/by-nd/2.0/ or send a letter to Creative Commons, 171 Second St, Suite 300, San Francisco, CA 94105, USA, or Eisenacher Strasse 2, 10777 Berlin, Germany 